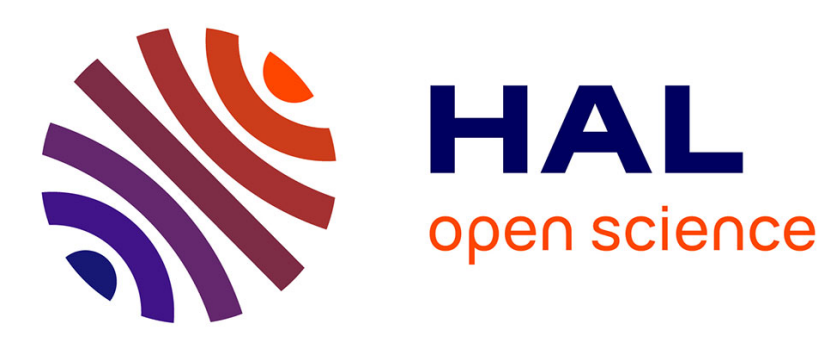

\title{
Implicit induction in conditional theories
}

Adel Bouhoula, Michaël Rusinowitch

\section{To cite this version:}

Adel Bouhoula, Michaël Rusinowitch. Implicit induction in conditional theories. [Research Report] RR-2045, INRIA. 1993. inria-00074627

\section{HAL Id: inria-00074627 https://hal.inria.fr/inria-00074627}

Submitted on 24 May 2006

HAL is a multi-disciplinary open access archive for the deposit and dissemination of scientific research documents, whether they are published or not. The documents may come from teaching and research institutions in France or abroad, or from public or private research centers.
L'archive ouverte pluridisciplinaire HAL, est destinée au dépôt et à la diffusion de documents scientifiques de niveau recherche, publiés ou non, émanant des établissements d'enseignement et de recherche français ou étrangers, des laboratoires publics ou privés. 


\section{Implicit Induction in Conditional Theories}

Adel BOUHOULA

Michaël RUSINOWITCH

\section{$\mathbf{N}^{\circ} 2045$}

Septembre 1993

PROGRAMME 2

Calcul symbolique, programmation

et génie logiciel

de recherche 


\title{
Implicit Induction in Conditional Theories ${ }^{* \dagger \ddagger}$
}

\author{
Adel Bouhoula and Michaël Rusinowitch \\ CRIN \& INRIA-Lorraine \\ BP 239, 54506 Vandoeuvre-lès-Nancy, France \\ email: \{bouhoula,rusi\}@loria.fr
}

September 18, 1993

\begin{abstract}
We propose a new procedure for proof by induction in conditional theories where case analysis is simulated by term rewriting. This technique reduces considerably the number of variables of a conjecture to be considered for applying induction schemes (inductive positions). Our procedure is presented as a set of inference rules whose correctness has been formally proved. Moreover, when the axioms are ground convergent it is possible to apply the system for refuting conjectures. The procedure is even refutationally complete for conditional equations with boolean preconditions over free constructors (under the same hypotheses). The method is entirely implemented in the prover SPIKE. This system has proved interesting examples in a completely automatic way, that is, without interaction with the user and without ad-hoc heuristics. It has also proved the challenging Gilbreath card trick, with only 2 easy lemmas.
\end{abstract}

keywords: Theorem proving, Term rewriting systems, Implicit induction, Conditional theories.

\section{Induction Implicite dans les théories Conditionnelles}

\section{Résumé}

On propose une nouvelle procédure de preuve par induction dans les théories conditionnelles où l'analyse par cas est simulée par la réécriture. Cette technique réduit considérablement le nombre des variables d'une conjecture à considérer pour l'application des schémas d'induction (positions inductives). Notre procédure est présentée par un ensemble de règles d'inférence dont la correction a été formellement prouvée. De plus, si les axiomes sont convergents sur les termes clos alors il est possible d'appliquer le système pour réfuter des conjectures non valides. La procédure est aussi réfutationnellement complète pour les équations conditionnelles avec préconditions booléennes sur des constructeurs libres (sous les mêmes hypothèses). Notre méthode est entièrement implémentée dans le prouveur SPIKE. Ce système a prouvé des exemples intéressants d'une manière automatique, i.e. sans interaction avec l'utilisateur et sans heuristiques. Par exemple, 2 lemmes suffisent pour prouver le tour de cartes de Gilbreath.

mots clés: Preuve automatique, Système de réécriture, Induction implicite, Théorie conditionnelles.

* This is an extended version of a paper presented at the International Joint Conference on Artificial Intelligence, Chambéry (France), 1993.

tWe thank our colleagues from the IndusMind group for stimulating discussions.

${ }^{\prime}$ Partly supported by the PRC mécanisation du raisonnement and the Esprit BRA workshop COMPASS 


\section{Introduction}

Formal methods are more and more frequently adopted by industry for hardware and software verification. They require efficient automatic tools to relieve designers and programmers of the related proof obligations. Mathematical induction is essential as a technique for building formal proofs in this context. Its power is expressed by the successes of Nqthm [Boyer and Moore, 1979] that has been for many years the only significant automated theorem proving system for induction. However $\mathrm{Nqthm}$ requires a lot of interaction with the user. For instance many lemmas need to be given to Nqthm as milestones even for simple proof tasks.

Another direction for automating induction was proposed in the early eighties, the inductionless induction technique [Musser, 1980; Huet and Hullot, 1982; Kapur and Musser, 1987] whose principle is to simulate induction by term rewriting. This method is refutational and does not require human interaction. While very limited at the beginning, its domain of application has widened considerably, thanks to the contributions of [Jouannaud and Kounalis, 1986] who relaxed the conditions on constructor symbols and of [Fribourg, 1986] who showed that only linear derivations were needed (see also [Bachmair, 1988]). More recently the method has been completely freed from the completion framework [Kounalis and Rusinowitch, 1990a; Reddy, 1990]. It has now become possible to apply it to conditional equational theories [Kounalis and Rusinowitch, 1990b; Bouhoula et al., 1992a]. Inductionless induction in our new setting reduces to firstly instanciating conjectures by induction schemes called test sets and secondly simplifying them by axioms, other conjectures or induction hypotheses. Every iteration generates new lemmas that are processed in the same way as the initial conjectures. The method does not require any hierarchy between the lemmas. They are all stored in a list and using conjectures for mutual simplification simulates simultaneous induction. The system SPIKE has been developed [Bouhoula et al., 1992b] on this principle and incorporates many optimizations such as powerful simplification techniques. To our knowledge, this system is the only one that can prove and disprove inductive theorems in conditional theories without any interaction.

However computer experiments have convinced us of the necessity of introducing a proper rule to perform case reasoning. Case analysis is a fundamental reasoning technique. A typical instance of it is the cut rule that consists in splitting a goal formula $A$ along another formula $C$ for generating two subgoals $C \Rightarrow A$ and $\neg C \Rightarrow A$. The main difficulty, already recognized by logicians a long time ago, relies in the choice of the cut formula $C$. A natural solution when dealing with theories axiomatized by Horn clauses (or conditional equations) is to use the negative literals of the axioms as cut formulas. This approach is frequently used in the context of conditional rewrite system when a conditional rule $C \Rightarrow l \rightarrow r$ may reduce a goal $A$ to subgoals $C \Rightarrow A[r / l]$ (i.e. term $l$ is replaced by $r$ in $A$ ) and $\neg C \Rightarrow A$.

The problem is now that one of the subgoals is not smaller than the initial goal and a lot of control is needed to avoid divergence of the process since a similar case analysis can be applied again to $\neg C \Rightarrow A$. This has motivated us to introduce a new case analysis rule that allows one to splitting a goal $A$ into subgoals $C_{i} \Rightarrow A\left[l_{i} / r_{i}\right]$ and $\vee_{i} C_{i}$. Since in the context of conditional rewrite system all subgoals are strictly smaller than the initial goal the search space is much more controlled. Related approaches were proposed independently by [Bronsard and Reddy, 1990] and [Bevers, 1993]. However, since their inference systems are unable to handle non Horn clauses, they cannot prove the $\vee_{i} C_{i}$ formulas otherwise than by external means. On the contrary, our proof technique applies to non Horn clauses as well. The disjunction $V_{i} C_{i}$ is added to the other conjectures and does not require particular treatment. Therefore our setting is very homogeneous and permits one to extend our basic inference rules by various optimizations without loosing correctness and completeness. In particular we have a notion of inductive positions defining the subset of variables of a conjecture that can be instanciated by induction schemes and we have proved that these positions are the only ones needed for completeness. The restriction of induction to these positions reduces drastically the search space. The importance of such restrictions was recognized a long time ago by [Boyer and Moore, 1979]. 
The paper is organized as follows. In Section 2 we introduce the basic definitions about term rewriting. In Section 3 we define the notions of inductive theory and inductive rewriting, which is a fundamental tool for proving inductive theorems. We define in section 4 inductive positions and test sets. Section 5 presents our technique of simulating case reasoning by rewriting. This technique reduces considerably the number of inductive positions to be considered. The strategy can be embedded in a correct set of inference rules described in Section 6 . When the axioms are ground convergent and the defined functions are completely defined then it is possible to apply the system for refuting conjectures (subsection 6.3). In Section 7 , the strategy is even proved refutationally complete for conditional equations with boolean preconditions (under previous hypotheses). Some optimizations are given in section 8 and computer experiments with SPIKE are discussed in Section 9.

SPIKE has proved the challenging Gilbreath card trick. The proof is based on $5(2$, with H. Zhang's formulation) lemmas that are easy to understand. This example was treated by $B$. Boyer and $\mathrm{H}$. Zhang. Unlike us, they require a lot of lemmas, some of them being non-obvious. In appendix, we give a detailed account of SPIKE's proof.

\section{Basic concepts}

We assume that the reader is familiar with the basic notions of rewrite systems. We introduce the essential terminology below and refer to [Dershowitz and Jouannaud, 1990] for more detailed presentations.

\subsection{Terms and substitutions}

A many-sorted signature $\Sigma$ is a pair $(S, F)$ where $S$ is a set of sorts and $F$ is a finite set of function symbols. We assume that we have a partition of $F$ in two subsets, the first one, $C$, contains the constructor symbols and the second, $D$, is the set of defined symbols.

Let $X$ be a family of free sorted variables and let $T(F, X)$ be the set of well-sorted $F$ terms. $\operatorname{Var}(t)$ stands for the set of all variables appearing in $t$ and $\sharp(x, t)$ denotes the number of occurrences of the variable $x$ in $t$. A variable $x$ in $t$ is linear iff $\sharp(x, t)=1$. If $\operatorname{Var}(t)$ is empty then $t$ is a ground term. By $T(F)$ we denote the set of all ground terms. From now on, we assume that there exists at least one ground term of each sort.

For any term $t, o c c(t) \subseteq N^{*}$ denotes its set of positions and the expression $t / u$ denotes the subterm of $t$ at a position $u$. The root position is written $\epsilon$. We write $t[s]_{u}$ (resp. $t[s]$ ) to indicate that $s$ is the subterm of $t$ at position $u$ (resp. at some position). Also let $t(u)$ denote the symbol of $t$ at position $u$. A position $u$ in a term $t$ is said to be $a$ strict position if $t(u)=f \in F$, a variable position if $t(u)=x \in X$ and $\sharp(x, t)=1$, a non-linear variable position if $t(u)=x \in X$ and $\sharp(x, t)>1$. We use $s d o m(t)$ to denote the set of strict positions in $t$. If $u$ is a position, then $|u|$ (the length of the corresponding string) gives us its depth.

A $\Sigma$ - substitution assigns $\Sigma$ - terms of appropriate sorts to variables. Composition of substitutions $\sigma$ and $\eta$ is written by $\sigma \eta$. The $\Sigma-$ term $t \eta$ obtained by applying a substitution $\eta$ to $t$ is called an instance of $t$. If $\eta$ is a ground substitution (i.e. $x \eta$ is ground for every $x$ ), we say that $t \eta$ is a ground instance of $t$.

\subsection{Conditional Equations and Clauses}

Let $\Sigma=(S, F)$ be a signature. A $\Sigma$-equation is a pair $e=e^{\prime}$ where $e, e^{\prime} \in T(F, X)$ are terms of the same sort. A conditional $\Sigma$-equation is either a $\Sigma$-equation or an expression of one of the following forms: $\left(e_{1} \wedge \ldots \wedge e_{n} \Rightarrow e\right)$, or $\left(e_{1} \wedge \ldots \wedge e_{n} \Rightarrow\right)$, or $(\Rightarrow)$ where $e, e_{1}, \ldots e_{n}$ are $\Sigma$-equations. $e_{1}, \ldots e_{n}$ are the conditions and $e$ is the conclusion. A $\Sigma$-clause is an expression of the form $\neg e_{1} \vee \neg e_{2} \vee \ldots \vee \neg e_{n} \vee e_{1}^{\prime} \vee \ldots \vee e_{m}^{\prime}$. When $\Sigma$ is clear from the context, we omit the prefix $\Sigma$. We identify a conditional equation and its corresponding representation as a 
Horn clause. A clause is positive if $\neg$ does not occur in it. Let $c_{1}$ and $c_{2}$ two clauses such that $c_{1} \sigma$ is a subclause of $c_{2}$ for some substitution $\sigma$, then we say that $c_{1}$ subsumes $c_{2}$.

In this paper, axioms are built from conditional equations and goals to be proved are clauses, i.e. disjunction of equational literals, since $=$ is the only predicate ${ }^{1}$. The symbol $\equiv$ is used for syntactic equality between two objects.

\subsection{Rewrite Relations}

\subsubsection{Preliminaries}

Given a binary relation $\rightarrow, \rightarrow^{*}$ denotes its reflexive and transitive closure. Let $a$ and $b$ be two terms, we write $a \downarrow b$, if there exists $c$ such that $a \rightarrow^{*} c$ and $b \rightarrow^{*} c$. A relation $\rightarrow$ is noetherian if there is no infinite sequence $t_{1} \rightarrow t_{2} \rightarrow \ldots$ In the following, we suppose given a reduction ordering $\succ$ on the set of terms, that is a transitive irreflexive relation that is noetherian, monotonic ( $s \succ t$ implies $w[s] \succ w[t])$ and stable $(s \succ t$ implies $s \sigma \succ t \sigma)$. We also assume that the ordering $\succ$ can be extended consistently when adding new constants to the signature. The multiset extension of an ordering $\succ$ will be denoted by $\gg$. Given a congruence relation $\approx$ on terms that is stable $(s \approx t$ implies $s \sigma \approx t \sigma)$ and compatible with $\succ\left(s \succ t, s \approx s^{\prime}, t \approx t^{\prime}\right.$ implies $s^{\prime} \succ t^{\prime}$ ) we define $\succeq$ as $\succ U \approx$. We write $s * t$ when $s \nsucceq t$ and $t \nsucceq s$.

A conditional equation $a_{1}=b_{1} \wedge \ldots a_{n}=b_{n} \Rightarrow s=t$ will be written as $a_{1}=b_{1} \wedge \ldots \wedge a_{n}=$ $b_{n} \Rightarrow s \rightarrow t$ if $\{s \sigma\} \gg\left\{t \sigma, a_{1} \sigma, b_{1} \sigma, \ldots, a_{n} \sigma, b_{n} \sigma\right\}$ for all ground substitutions $\sigma$; in that case we say that $a_{1}=b_{1} \wedge \ldots a_{n}=b_{n} \Rightarrow s \rightarrow t$ is a conditional rule. The term $s$ is the left-hand side of the rule. A set of conditional rules is a rewrite system.

\subsubsection{Conditional Rewriting}

The idea of rewriting is to impose a direction when using equations in proofs. This direction is indicated by an arrow when it is independent from the instantiation: $l \rightarrow r$ means that we can replace $l$ by $r$ in any context. When an instance of a conditional equation is orientable and has a valid conditional part it can be applied as a rule. The conditions are checked recursively. Termination is ensured by requiring the conditions to be smaller (w.r.t. the reduction ordering $\succ$ ) than the conclusion.

Definition 2.1 (Conditional Rewriting) Let $R$ be a set of conditional equations. Let a be a term and $u$ a position in $a$. We write: $a[s \sigma]_{u} \rightarrow_{R} a[t \sigma]_{u}$ if there is a substitution $\sigma$ and $a$ conditional equation $\wedge_{i=1}^{n} a_{i}=b_{i} \Rightarrow s=t$ in $R$ such that:

1. $s \sigma \succ t \sigma$.

2. $\forall i \in[1 . . n] a_{i} \sigma \downarrow_{R} b_{i} \sigma$.

3. $\left\{a[s \sigma]_{u}\right\} \gg\left\{a_{1} \sigma, b_{1} \sigma, \ldots, a_{n} \sigma, b_{n} \sigma\right\}$.

A term $t$ is reducible w.r.t. to $\rightarrow_{R}$ if there is a term $t^{\prime}$ such that $t \rightarrow_{R} t^{\prime}$. Otherwise we say $\mathrm{t}$ is $R$-irreducible. The system $R$ will be qualified as ground convergent if for all $a, b$ in $T(F)$

$$
R \vDash a=b \text { implies } a \downarrow_{R} b
$$

Note that when $R$ is a rewrite system the relation $\rightarrow_{R}$ is similar to the notion of decreasing rewriting of Dershowitz, Okada and Sivakumar [Dershowitz et al., 1988].

\footnotetext{
${ }^{1}$ It is straightforward to extend the following results to signatures with other predicates than equality.
} 


\subsubsection{Sufficient completeness}

When for all possible arguments the result of a defined operator can be expressed with constructors only, we say that this operator is completely defined w.r.t. the constructors. This requirement is very natural when building specifications in a structured way [Guttag, 1978]. Here is a more formal definition:

Definition 2.2 Let $R$ be a rewrite system, let $C$ be a set of constructors and $D$ be a set of defined operator. The operator $f \in D$ is completely defined w.r.t. $C$ iff for all $t_{1}, \ldots, t_{n}$ in $T(C)$, there exists $t$ in $T(C)$ such that $f\left(t_{1}, \ldots, t_{n}\right) \rightarrow_{R}^{*} t$.

Although this property is in general undecidable, our system SPIKE offers facilities to check and complete definitions. The program builds a pattern tree for every defined operator $f$ (see [Kounalis, 1990]). The leaves of the tree give a partition of the possible arguments for $f$. Then if all the leaves are reducible, the answer is affirmative. If one of the leaves is not reducible then SPIKE suggests a new rule for completing the specification. This rule is not entirely determined but rather a possible schema for it is proposed, namely: (condition, left-hand-side). Once the user has chosen the new rule, usually by simply giving its right-hand side, SPIKE replays the test.

Example 2.1 Consider the specification of lists of natural numbers with an "insert" operation and a "sorted" predicate on lists that is true iff a list is ordered. We have $C=\{0, s$, True, False, cons, $N i l\}$ and $D=\{\leq$, sorted, insert $\}$.

$$
\begin{aligned}
\text { True }=\text { False } & \Rightarrow \\
0 \leq x & \rightarrow \text { True } \\
s(x) \leq 0 & \rightarrow \text { False } \\
s(x) \leq s(y) & \rightarrow x \leq y \\
\operatorname{sorted}(N i l) & \rightarrow \text { True } \\
\operatorname{sorted}(\operatorname{cons}(x, N i l)) & \rightarrow \text { True } \\
x \leq y=\text { False } \Rightarrow \operatorname{sorted}(\operatorname{cons}(x, \operatorname{cons}(y, z))) & \rightarrow \text { False } \\
x \leq y=\text { True } \Rightarrow \operatorname{sorted}(\operatorname{cons}(x, \operatorname{cons}(y, z))) & \rightarrow \operatorname{sorted}(\operatorname{cons}(y, z)) \\
\text { insert }(x, N i l) & \rightarrow \operatorname{cons}(x, N i l) \\
x \leq y=\text { True } \Rightarrow \text { insert }(x, \operatorname{cons}(y, z)) & \rightarrow \operatorname{cons}(x, \operatorname{cons}(y, z)) \\
x \leq y=\text { False } \Rightarrow \text { insert }(x, \operatorname{cons}(y, z)) & \rightarrow \operatorname{cons}(y, \text { insert }(x, z))
\end{aligned}
$$

All defined symbols are completely defined:

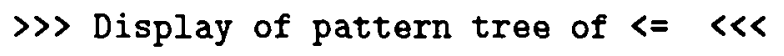

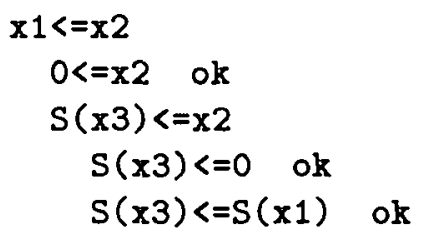




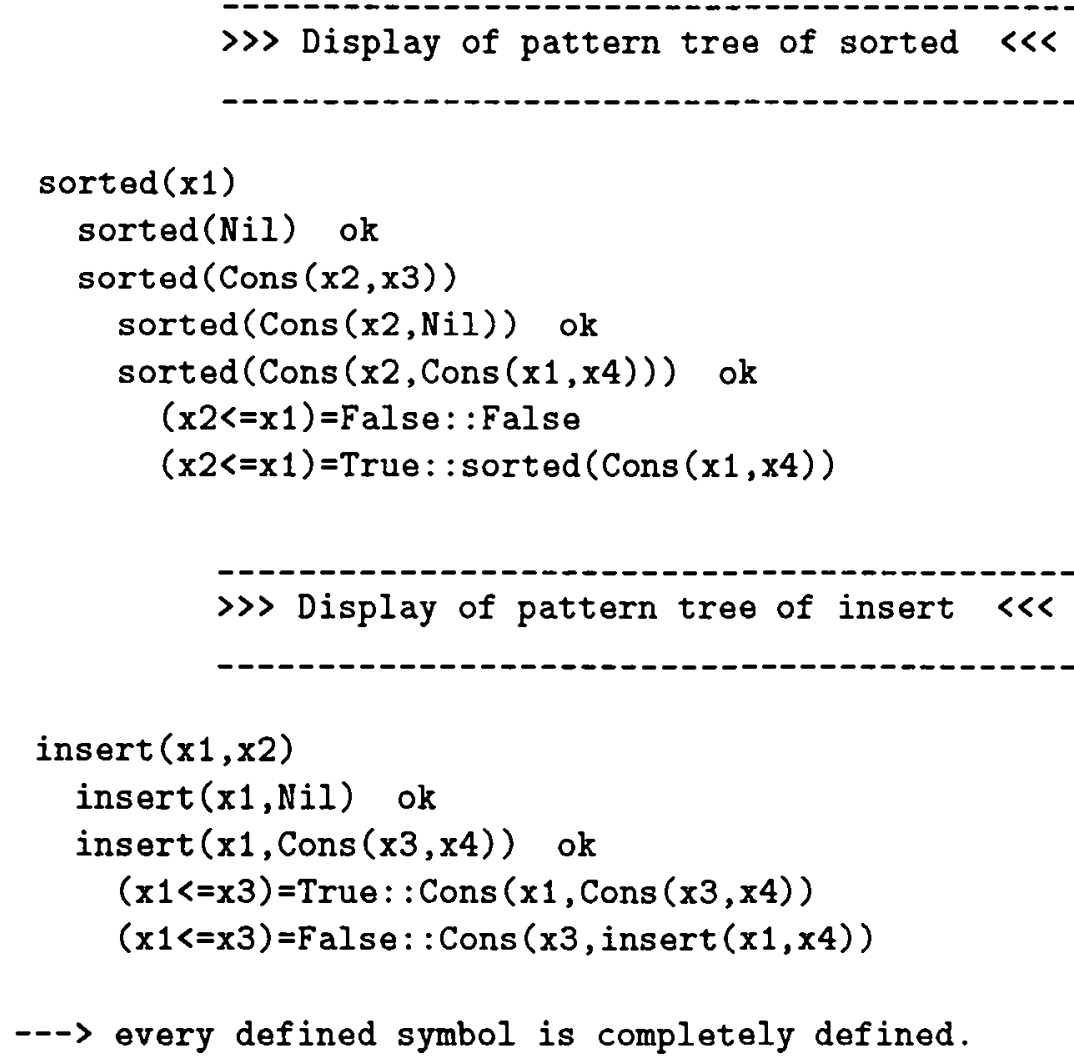

\section{Induction}

\subsection{Inductive theory}

Given a set of Horn clauses $A x$ on the signature $\Sigma$, we recall that a Herbrand (or term-generated) model of $A x$ is a model of $A x$ whose domain is the set of ground terms (axioms for equality are implicitly assumed to be valid, too). A formula $\mathcal{F}$ is a deductive theorem of $A x$ if it is valid in any model of $A x$. This will be denoted by $A x \vDash \mathcal{F}$. Deductive theorems can be proved by refutation, by deriving a contradiction from $\neg \mathcal{F} \wedge A x$. Usually $\neg \mathcal{F}$ is transformed into a universal sentence $\mathcal{U}$ by introducing skolem functions. Hence the signature $\Sigma$ has to be extended.

The theory of $A x$, which is the one we are interested in, is the class of sentences that are true in the minimal Herbrand (or initial) model of $A x$. For detailed definitions of initial models see, for instance, [Padawitz, 1988]. Every element in the domain of a Herbrand model is denoted by a ground term built on the signature of $A x$. But since ground terms can easily be well-ordered, induction is available as a natural technique to prove sentences in these models. This is why we shall call inductive theory of $A x$ the class of sentences that are valid in the minimal Herbrand model of $A x$ :

Definition 3.1 Let Ax be a set of Horn clauses on the signature $\Sigma$. A clause $e$ is an inductive consequence of $A x$ if it is valid in the minimal Herbrand model of Ax. This will be denoted by $A x \models_{\text {ind }} e$.

The following proposition gives us a useful characterization of inductive consequences:

Proposition 3.1 A clause $\neg C_{1} \vee \ldots \vee \neg C_{n} \vee P_{1} \vee \ldots \vee P_{m}$ is an inductive consequence of $A x$ if and only if for any ground substitution $\sigma$,

$$
\text { (for all } i: A x \models C_{i} \sigma \text { ) implies (there exists } j \text { such that } A x \models P_{j} \sigma \text { ) }
$$


For clauses validity in all Herbrand models differs, in general, from validity in the initial model. However, these two notions of validity coincide for unconditional equations [Padawitz, 1988].

\subsection{Inductive rewriting}

To simplify goals, we extend the conditional rewriting relation definition 2.1 , so that we can check the conditions of a rule to be applied to a clause $C$ with inductive hypothesis, other conjectures and the premisses of $C$, considered as an implication formula.

Let us first introduce a few notations. Let $C \equiv \neg\left(a_{1}=b_{1}\right) \vee \ldots \vee \neg\left(a_{n}=b_{n}\right) \vee\left(c_{1}=\right.$ $\left.d_{1}\right) \vee \ldots \vee\left(c_{m}=d_{m}\right)$. Then we denote by $\operatorname{prem}(C)$ the set of negated atoms of $C:\left\{a_{i}=b_{i}\right\}_{i=1, n}$. The expression $(a=b)^{\varepsilon}$ denotes the literal $a=b$ if $\varepsilon=+$ and the literal $\neg(a=b)$ if $\varepsilon=-$. The skolemized clause $\bar{C}$ of $C$ is the clause obtained by substituting every variable of $C$ by a new constant. We recall that $\succ$ can be extended consistently to terms with new symbols.

Definition 3.2 (Inductive rewriting) Let $R$ and $W$ be two sets of conditional equations. Consider a clause $C \equiv(a=b)^{\epsilon} \vee r$ and its skolemized version $\bar{C} \equiv(\bar{a}=\bar{b})^{\varepsilon} \vee \bar{r}$. We write: $a \mapsto R(W] ; r a^{\prime}$ if $a \succ a^{\prime}$ and:

either $\bar{a} \mapsto_{\text {prem }(\bar{r})} \overline{a^{\prime}}$

or there exists a position $u$ in $a, a$ substitution $\sigma$ and a conditional equation $\wedge_{i=1}^{n} a_{i}=b_{i} \Rightarrow s=t$ in $R$ such that:

1. $a \equiv a[s \sigma]_{u}$ and $a^{\prime} \equiv a[t \sigma]_{u}$.

2. $\left\{a[s \sigma]_{u}\right\} \gg\left\{a_{1} \sigma, b_{1} \sigma, \ldots, a_{n} \sigma, b_{n} \sigma\right\}$.

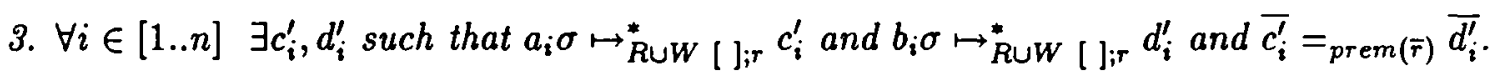
where $=_{\text {prem }(\bar{r})}$ is the congruence generated by prem $(\bar{r})$.

The set $W$ in the definition is intended to contain induction hypotheses in the proof system described below. Inductive rewriting can be viewed as a generalization of both the rewriting relation defined in [Kounalis and Rusinowitch, 1990b] and contextual rewriting [Zhang, 1993].

Example 3.1 With $R$ as in example 2.1, here is an example of a clause which is inductively rewritten by its own conditions:

Simplification of:

$s(x 2)<x 3=$ False $\Rightarrow$

$x 1<x 2=$ True, $s(x 1)<x 3=\operatorname{True}$, False $=\operatorname{sorted}(\operatorname{Cons}(s(s(x 2)), \operatorname{Cons}(s(x 3), N i 1)))$

by $R[] ; r$ :

$s(x 2)<x 3=$ False $\Rightarrow x 1<x 2=$ True, $s(x 1)<x 3=$ True, False $=$ False

In this example we have:

$$
\text { sorted }(\text { Cons }(s(s(x 2)), \text { Cons }(s(x 3), N i l))) \mapsto R[\text { ]; }\{s(x 2)<x 3=F \text { alse }\} \text { False }
$$

Example 3.2 The following rules define odd and even for nonnegative integers:

$$
\begin{aligned}
\text { even }(0) & \rightarrow \text { True } \\
\text { even }(s(0)) & \rightarrow \text { False } \\
\text { even }(s(s(x))) & \rightarrow \text { even }(x) \\
\text { even }(x)=\text { True } \Rightarrow \text { odd }(x) & \rightarrow \text { False } \\
\text { even }(x)=\text { False } \Rightarrow \text { odd }(x) & \rightarrow \text { True } \\
\text { True }=\text { False } \Rightarrow &
\end{aligned}
$$

We consider the following conjectures ( + is defined as usual): 
$E O=\{\operatorname{odd}(s(x+x))=\operatorname{Tru} \theta, \operatorname{odd}(x+x)=F a l s e, \operatorname{even}(s(x+x))=F$ alse, even $(x+x)=$ True $\}$

We show an example where a clause is inductively rewritten with a conjecture from the initial set $E_{0}$ (H0 the initial set of inductive hypothesis is empty):

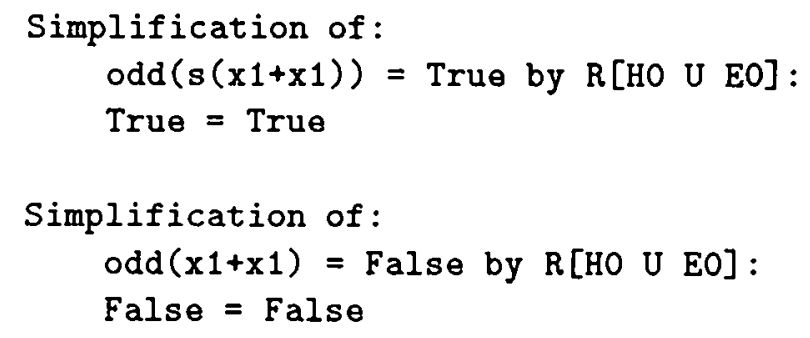

Remark that inductive rewriting possesses a kind of stability in the sense that for any term $s, t$, any clause $r$ and substitution $\tau, s \mapsto_{R[W] ; r} t$ implies $s \tau \mapsto_{R[W] ; r \tau} t \tau$. If $W$ is empty, $\mapsto_{R[W] ; r}$ will be denoted by $\mapsto R ; r$.

Lemma 1 For all substitutions $\tau: s \mapsto_{R[W] ; r} t$ implies $s \tau \mapsto_{R[W] ; r \tau} t \tau$.

proof: We have two cases:

1. $W=\emptyset$ then a proof is done by noetherian induction on $s$ with $\succ$. We have $s \succ t$ then $s \tau \succ t \tau$. Now, we consider two cases:

(a) $s \mapsto_{R ; r} t$ with $\bar{s} \mapsto_{\text {prem }(\bar{r})} \bar{t}$, then $\overline{s \tau} \mapsto_{\text {prem }(\overline{r \tau})} \overline{t \tau}$.

(b) There exists a conditional equation $\wedge_{i} a_{i}=b_{i} \Rightarrow l=r$ of $R$ such that:

i. $s / u=l \sigma$ for a position $u$ in $s$ and a substitution $\sigma$, then we have also: $s \tau / u=l \sigma \tau$.

ii. $t=s[r \sigma]_{u}$, clearly: $t \tau=s \tau[r \sigma \tau]_{u}$.

iii. $\{a\} \gg\left\{a_{1} \sigma, \ldots, b_{n} \sigma\right\}$ implies that $\{a \tau\} \gg\left\{a_{1} \sigma \tau, \ldots, b_{n} \sigma \tau\right\}$.

iv. $\forall i \in[1 \ldots n]$ :
A. $a_{i} \sigma \mapsto{ }_{R ; r}^{*} c_{i}^{\prime}$, since $s \succ a_{i} \sigma$, by the induction hypothesis: $a_{i} \sigma \tau \mapsto_{R ; \tau \tau}^{*} c_{i}^{\prime} \tau$.
B. $b_{i} \sigma \mapsto{ }_{R ; r}^{*} d_{i}^{\prime}$, since $s \succ b_{i} \sigma$, by the induction hypothesis: $b_{i} \sigma \tau \mapsto_{R ; r \tau}^{*} d_{i}^{\prime} \tau$.
C. $\overline{c_{i}^{\prime}}={ }_{\text {prem }(\bar{r})} \overline{d_{i}^{\prime}}$ which implies: $\overline{c_{i}^{\prime} \tau}={ }_{\text {prem }(\bar{r} \bar{r})} \overline{d_{i}^{\prime} \tau}$.

All this implies: $s \tau \mapsto R ; r \tau t \tau$.

2. $W \neq \emptyset$. Using 1 . the proof is obvious.

\section{Selection of Induction Schemes}

To perform a proof by induction, it is necessary to provide some induction schemes. In our framework these schemes are defined first by a function which, given a conjecture, selects the positions of variables where induction will be applied and second by a special set of terms called a test set. In general the selection of good inductive positions leads to drastic improvements.

Let us consider first the problem of choosing the positions where variables need to be instanciated by induction schemes. In order to define the set of these variables, we introduce $V L(s)$, the set of linear variables of a term $s$ and $D e f(f)$, the set of terms with root symbol $f$.

Definition 4.1 Given a rewrite system $R$ on $T(F, X)$ the set of inductive positions for a function symbol $f$ is: Occ_ind $(R, f)=\{u /$ there exists $p \Rightarrow g \rightarrow d \in R$ such that $g \in \operatorname{Def}(f), u \in$ $O c c(g) \backslash\{\epsilon\}$ and $g / u \notin V L(g)\}$. 
We say a variable $x$ is nullary if there are only finitely many ground constructor terms with the same sort as $x^{2}$. Given a term $s$, an induction variable of $s$ is a variable that is nullary or that occurs at a position $u . v$ of $s$ such that $v$ is an inductive position of the root of $s / u$ (if $s$ is a variable then it is considered as an induction variable). Given a term $s$ and a set of terms $T S, \sigma$ is a $T S$-substitution for $s$, if it maps any induction variable of $s$ to an element of $T S$ of the same sort and any other variable to itself.

A rewrite rule $c \Rightarrow s \rightarrow r$ is left-linear if $s$ is linear. A rewrite system $R$ is left-linear if every rule in $R$ is left-linear, otherwise $R$ is said to be non-left-linear. A term is strongly $R$-irreducible if none of its non-variable subterms matches a left-hand side of $R$. If $t$ is a term, then the depth of $t$ is the maximum of the depths of the positions in $t$ and denoted depth(t) or abusively $|t|$. The strict depth of $t$, written as sdepth(t), is the maximum of the depths of the strict positions in $t$. The depth of a rewrite system $R$, denoted $\operatorname{depth}(R)$, is defined as the maximum of the depths of the left-hand sides of $R$. Similarly, the strict depth of $R$, written a $\operatorname{sdepth}(R)$, is the maximum of the depths of the strict positions in the left-hand sides of $R$. We define the number $D(R)$ to be $\operatorname{depth}(R)-1$ if $\operatorname{sdepth}(R)<\operatorname{depth}(R)$ and $R$ is left linear, $\operatorname{depth}(R)$ otherwise.

Instead of mapping induction variables to any terms we plan to instanciate them by some well-chosen elements that we call test-sets. A definition of test-sets for conditional theories was introduced in [Bouhoula et al., 1992a]. It is possible to compute test sets for equational theories (see [Kounalis, 1990; Huber, 1991]). Unfortunately no algorithm exists for the general case of conditional theories.

Here we give a simpler definition of test-sets that will be sufficient for our purpose (we take advantage of the separation of symbols between defined and constructor operators).

Definition 4.2 If $R$ is a set of conditional rules then a test-set $S(R)$ for $R$ is a finite set of $R$-irreducible constructor terms that has the following properties:

a. for any $R$-irreducible ground term $s$ there exists a term $t$ in $S(R)$ and a ground substitution $\sigma$ such that $t \sigma=s$;

b. any non-ground term in $S(R)$ admits only non nullary variables and they may occur only at depth greater or equal than $D(R)$.

The first property allows us to prove theorems by induction on the domain of irreducible terms rather than on the whole set of terms. Sets of terms with the property a. are usually called cover sets in the literature [Reddy, 1990; Zhang et al., 1988]. However they cannot be used to refute theorems. The second property $b$. of test sets is fundamental for this purpose. It ensures that when an instance of a clause by an $S(R)$-substitution does not match any left-hand side of $R$ this reveals an inconsistency (under some additional conditions to be detailed later).

The properties of test-sets that we shall need later on are listed in the next proposition.

Proposition 4.1 We assume here that the constructors are free. For any term $t$ and $\sigma, S(R)$ substitution for $t$ we have:

a. $t \sigma$ does not contain an induction variable.

b. if $t \sigma$ is strongly $R$-irreducible then there exists $\tau$ such that $t \sigma \tau$ is ground and strongly $R$ irreducible.

proof:

a. Without loss of generality, we can assume that $t$ is a term $f\left(t_{1}, \ldots, t_{n}\right)$ where $f$ is a defined operator and the $t_{i}$ are elements of $T(C, X)$. Let $l$ be depth $(t)$ and let $x$ be a variable in $t \sigma$ at occurrence $u$. We can assume that $x$ does not occur in $t$, then by definition of test-sets we have $|u|>D(R)$. There are two cases to consider:

\footnotetext{
${ }^{2}$ this property can easily be decided when the constructors are free
} 
- if $R$ is left-linear and $\operatorname{sdepth}(R)<\operatorname{depth}(R)$. Then $|u| \geq \operatorname{depth}(R)$. If $u$ is an inductive position of $f$ then there is a left-hand side of a rule where a function symbol occurs at position $u$. Since $\operatorname{sdepth}(R)<\operatorname{depth}(R)$ there is a rule whose left-hand side $g$ satisfies $\operatorname{depth}(g)>|u|$ and this contradicts $|u| \geq \operatorname{depth}(R)$. Hence $x$ cannot be an inductive variable of $t \sigma$.

- otherwise $|u|>$ depth $(R)$. Hence $u$ cannot be an occurrence of a left-hand side of a rule.

b. It is enough to prove the Claim: let $t$ be a strongly $R$-irreducible term that does not contain any induction variable then there exists a ground instance $t \phi$ that is strongly $R$-irreducible. Assume that $\operatorname{Var}(t)=\left\{x_{1}, \ldots, x_{k}\right\}$ and consider a ground substitution $\phi$ such that any $\phi(x)$ is strongly $R$-irreducible and:

$$
\begin{aligned}
& \text { a. } \forall i \in[1 \ldots k],\left|x_{i} \phi\right|>|t| \\
& \text { b. } \forall i, j \in[1 \ldots k], i \neq j, \quad \| x_{i} \phi|-| x_{j} \phi||>|t|
\end{aligned}
$$

Since the variables $x_{i}$ are not nullary it is possible to build such a substitution by choosing the $\phi\left(x_{i}\right)$ among the terms built from constructor symbols only. Assume now that $t \phi$ contains an instance of a left-hand side $g$ of a rule in $R$. Now, since any $\phi\left(x_{i}\right)$ is strongly $R$-irreducible, there is a strict position $u$ in $t$ such that $t / u$ is an instance of $g$. Let $v$ be a position of $g$ such that $g / v$ is a function symbol. $t / u v$ is a function symbol since $t$ does not contain any induction variable. We consider two cases:

- Assume that $g$ is linear. We can define a substitution $\sigma$ such that for every variable $x$ that occurs at position $w$ of $g$ we have $\sigma(x)=t / u w$. Such a substitution exists by linearity of $g$. We then have $t / u=g \sigma$ which is a contradiction with the assumption that $t$ is strongly $R$-irreducible.

- Assume that $g$ is non linear. Since $t$ is not an instance of $g$ (and $t / u w=g / w$ for every strict position $w$ of $g$ ) there exist two occurrences $u_{1}$ and $u_{2}$ of a variable $x$ in $g$ such that: $t / u u_{1} \neq t / u u_{2}$ and $t \phi / u u_{1}=t \phi / u u_{2}$. There are three cases to be considered:

a. if $t / u u_{1}$ and $t / u u_{2}$ are ground. In this case $t / u u_{1}=t \phi / u u_{1}$ and $t / u u_{2}=t \phi / u u_{2}$. Therefore $t / u u_{1}=t / u u_{2}$, contradiction.

b. if $t / u u_{1}$ is ground and $t / u u_{2}$ non ground. Then some $x_{i}$ occurs in $t / u u_{2}$. We have $\left|x_{i} \phi\right|>|t|$ by construction of $\phi$ and therefore $\left|t \phi / u u_{2}\right|>|t|$. On the other hand $\left|t \phi / u u_{2}\right|=\left|t \phi / u u_{1}\right|=\left|t / u u_{1}\right| \leq|t|$. Contradiction.

c. if $t / u u_{1}$ and $t / u u_{2}$ are non ground. Then there is an occurrence $v$ and a variable $x_{k}$ such that $t / u u_{1} v=x_{k}$ and $t / u u_{2} v \neq x_{k}$. If $t / u u_{2} v$ is ground the proof is similar to $b .$. If $t / u u_{2} v$ is non ground let $\operatorname{Var}\left(t / u u_{2} v\right)=\left\{x_{i_{1}}, \ldots, x_{i_{m}}\right\}$. If $x_{k} \in \operatorname{Var}\left(t / u u_{2} v\right)$ then $\left|t \phi / u u_{1} v\right|<\left|t \phi / u u_{2} v\right|$ and therefore we cannot have $t \phi / u u_{1}=t \phi / u u_{2}$, contradiction. If $x_{k} \notin \operatorname{Var}\left(t / u u_{2} v\right)$ then let $x_{j}$ be the variable in $\operatorname{Var}\left(t / u u_{2} v\right)$ such that $\left|x_{j} \phi\right|=$ $\max _{l=1, \ldots, m} \mid x_{j_{l}} \phi$.

If $\left|x_{k} \phi\right|>\left|x_{j} \phi\right|+|t|$ then $\left|t \phi / u u_{1} v\right|=\left|x_{k} \phi\right|>\left|x_{j} \phi\right|+|t|>\left|t \phi / u u_{2} v\right|$ If $\left|x_{j} \phi\right|>$ $\left|x_{k} \phi\right|+|t|$ then $\left|t \phi / u u_{2} v\right| \geq\left|x_{j} \phi\right|>\left|x_{k} \phi\right|+|t|>\left|t \phi / u u_{1} v\right|=\left|x_{k} \phi\right|$ and we derive a contradiction, too.

It is easy to compute test-sets in the case where all functions are completely defined:

Proposition 4.2 If all defined operators are completely defined over free constructors then the set $T$ of constructor terms (up to variable renaming) of depth $\leq D(R)$ where not nullary variables may occur only at depth $D(R)$ is a test-set for $R$. 
proof: since the defined operators are completely defined any ground irreducible terms is built only with constructors and therefore is an instance of an element of $T$. The second property of the definition is trivially verified.

The role of test sets for refutation is shown by the following definition that gives a falsity criteria for positive clauses:

Definition 4.3 Suppose that we are given a rewrite system $R$ and a test set $S(R)$. Then a clause $C \equiv g_{1}=d_{1} \vee \ldots \vee g_{n}=d_{n}$ is quasi-inconsistent with respect to $R$ if there is a $S(R)$-substitution $\sigma$ of $C$ such that for all $1 \leq j \leq n, g_{j} \sigma \not \equiv d_{j} \sigma$ and the maximal elements of $\left\{g_{j} \sigma, d_{j} \sigma\right\}$ w.r.t. $\succ$ are strongly $R$-irreducible.

The next theorem is analogous to one from [Kounalis and Rusinowitch, 1990a].

Theorem 4.1 Let $R$ be a ground convergent rewrite system with free constructors. If a positive clause $C$ is quasi-inconsistent, then $C$ is not an inductive consequence of $R$.

proof: it is a direct consequence of proposition 4.1 .

The following example was suggested by E. Kounalis:

Example 4.1 Consider the "union" operator defined on lists of natural numbers built with constructors $\{$ cons, Nil\}:

$$
\begin{aligned}
\text { union }(N i l, N i l) & \rightarrow \text { Nil } \\
\text { union }(\operatorname{cons}(x, N i l), N i l) & \rightarrow \operatorname{cons}(x, N i l) \\
\text { union }(N i l, \operatorname{cons}(x, l)) & \rightarrow \operatorname{cons}(x, l) \\
\text { union }\left(\operatorname{cons}(x, l), \operatorname{cons}\left(y, l^{\prime}\right)\right) & \rightarrow \operatorname{cons}\left(x, \operatorname{cons}\left(y, \text { union }\left(l, l^{\prime}\right)\right)\right)
\end{aligned}
$$

$R$ is ground convergent and $S(R)=\{N i l$, cons $(x, l), 0, s(x)\}$. Consider the conjecture union $\left(l, l^{\prime}\right)$ $=$ union $\left(l^{\prime}, l\right)$. Among the test instances, we have: union $\left(\operatorname{cons}(x, l), \operatorname{cons}\left(y, l^{\prime}\right)\right)=$ union $(\operatorname{cons}(y$, $\left.l^{\prime}\right)$, cons $\left.(x, l)\right)$ which simplifies to $\operatorname{cons}\left(x, \operatorname{cons}\left(y\right.\right.$, union $\left.\left.\left(l, l^{\prime}\right)\right)\right)=\operatorname{cons}\left(y, \operatorname{cons}\left(x\right.\right.$, union $\left.\left.\left(l, l^{\prime}\right)\right)\right)$. Among the test instances of this last conjecture we have: $\operatorname{cons}(x, \operatorname{cons}(y$, union $(N i l, N i l)))=$ $\operatorname{cons}(y, \operatorname{cons}(x$, union $(N i l, N i l)))$ which simplifies to $\operatorname{cons}(x, \operatorname{cons}(y, N i l))=\operatorname{cons}(y, \operatorname{cons}(x$, $N i l)$ ), which is quasi-inconsistent (Both members are distinct, $R$-irreducible and do not contain any induction variable). So union $\left(l, l^{\prime}\right)=u$ union $\left(l^{\prime}, l\right)$ is not an inductive consequence of $R$. Note that with test sets, we can easily refute false conjectures with variables.

\section{Automatic Case Analysis}

We shall introduce now the Case rewriting relation that allows one to reduce goals with conditional rules without attempting to check their preconditions. The preconditions are appended to the goal as a context. Case rewriting can be viewed as an implementation of case analysis that is well adapted to the given conditional axioms. We have found this rule absolutely necessary for proving non trivial conjectures with our automatic system. Moreover it is the basis of a refutationally complete system for boolean systems. In this section we first discuss the problems with a former definition of case rewriting. Then we propose a new definition of case rewriting that is easier to automate and has given much better results on experiments. A first version of case rewriting was proposed in [Kounalis and Rusinowitch, 1990b]. Given a term $l$ and a rule $p \Rightarrow g \rightarrow d$ such that $g$ matches a subterm of $l$ with substitution $\sigma$, this rewriting is expressed by the following inference rule:

$$
l[g \sigma]_{u} \vee r \vdash l[d \sigma]_{u} \vee \neg p \sigma \vee r, l[g \sigma]_{u} \vee p \sigma \vee r
$$


However an important control problem with this technique lies in the choice of the rule to apply during the proof by induction. This can be illustrated by the next example.

Example 5.1 With $R$ as in example 3.1. A test set here is $\{0, s(0), s(s(x))$, True, False $\}$. Let us prove:

$$
\text { even }(s(x))=\text { True } \vee \text { odd }(x)=\text { False }
$$

by the case rewriting rule above. Clause 18 can be split according to axiom 15 in the two following clauses:

$$
\begin{gathered}
\text { even }(x)=\text { True } \Rightarrow \text { even }(s(x))=\text { True } \vee \text { False }=\text { False } \\
\neg(\text { even }(x)=\text { True }) \Rightarrow \text { even }(s(x))=\text { True } \vee \text { odd }(x)=\text { False }
\end{gathered}
$$

19 is a tautology and 20 is equivalent to:

$$
\text { even }(x)=\operatorname{True} \vee \text { even }(s(x))=\text { True } \vee \text { odd }(x)=\text { False }
$$

Now splitting clause 21 with axiom 16 yields:

$$
\begin{gathered}
\text { even }(x)=\text { False } \Rightarrow \text { even }(x)=\text { True } \vee \text { even }(s(x))=\text { True } \vee \text { True }=\text { False } \\
\neg(\text { even }(x)=\text { False }) \Rightarrow \text { even }(x)=\text { True } \vee \text { even }(s(x))=\text { True } \vee \text { odd }(x)=\text { False }
\end{gathered}
$$

22 simplifies to:

$$
\text { even }(x)=\text { False } \Rightarrow \text { even }(x)=\text { True } \vee \text { even }(s(x))=\text { True }
$$

that can be proved an inductive consequence of $R$. Clause 23 is equivalent to:

$$
\text { even }(x)=\text { False } \vee \text { even }(x)=\text { True } \vee \text { even }(s(x))=\text { True } \vee \text { odd }(x)=\text { False }
$$

Note that the same case analysis can be applied infinitely often to 25 . A possible way to avoid divergence is to limit application of the case analysis rule. Case rewriting in [Kounalis and Rusinowitch, 1990b] is controlled by conditions for avoiding infinite applications of the same rule. However, even when adding these technical conditions the proof of 25 diverges.

These problems motivated us to introduce a new case rewriting technique that rewrites a term $t$ simultaneously to several terms $t_{1}, \ldots, t_{n}$ each reduction being respectively valid in different contexts $c_{1}, \ldots, c_{n}$. In other words, given a term $t$, we consider all the ways to rewrite it w.r.t. to axioms and positions. We must then prove that the disjunction $D P$ of the conditions of the applied rules is inductively valid. Note that $D P$ is usually a non Horn clause. Our approach to inductive proofs is non hierarchical: we can prove $D P$ by simply adding it to the set of conjectures to be further processed. In the following definition $C N F$ is a function that returns a conjunctive normal form of a universal formula. For instance, $C N F(P(x) \wedge Q(x))=\{P(x), Q(x)\}$.

Definition 5.1 (Case rewriting) Let $R$ be a rewrite system and $C \equiv(a=b)^{c} \vee r$ be a clause. We define $G$ as the set $\left\{\left\langle a[d \sigma]_{u}, P \sigma\right\rangle\right.$; there exists $P \Rightarrow g \rightarrow d$ in $R$, a position $u$ in a such that $a / u=g \sigma, g \sigma$ is $R$-irreducible and does not contain an inductive variable $\}$. Then Case_rewriting $\left((a=b)^{\varepsilon}, r\right)$ is the following set of clauses:

$$
\left\{\neg P \vee\left(a^{\prime}=b\right)^{\varepsilon} \vee r ;\left\langle a^{\prime}, P>\in G\right\} \cup C N F\left(\bigvee_{\left\langle a^{\prime}, P>\in G\right.} P\right)\right.
$$

Case rewriting can be applied with lemmas too. Note also that the conditions on $g \sigma$ in the definition of $G$ are only required for the completeness of the procedure. 
Example 5.2 (example 5.1 continued) With our method, the proof of 18 is as follows: we apply case rewriting to get:

$$
\begin{gathered}
\text { even }(x)=\text { True } \Rightarrow \text { even }(s(x))=\text { True } \vee \text { False }=\text { False } \\
\text { even }(x)=\text { False } \Rightarrow \text { even }(s(x))=\text { True } \vee \text { True }=\text { False }
\end{gathered}
$$

We must prove:

$$
\operatorname{even}(x)=\text { True } \vee \text { even }(x)=\text { False }
$$

26 is a tautology, 27 is simplified by $R$ into:

$$
\operatorname{even}(x)=\text { False } \Rightarrow \operatorname{even}(s(x))=\text { True }
$$

Instanciating $x$ in 29 by elements 0 and $s(0)$ from the test set, yields clauses that are simplified by $R$ and subsumed by an axiom. The instance of $x$ in 29 by $s(s(y))$ gives a clause that is simplified by $R$ and subsumed by 29 , which becomes an induction hypothesis. In the same way 28 can easily be proved.

Example 5.3 Consider the system $R$ :

$$
\begin{aligned}
p(x, 0, z) & \rightarrow \text { True } \\
p(x, s(y), z) & \rightarrow p(x, y, z) \\
p(y, x, z)=\text { True } \Rightarrow f(x, y, z) & \rightarrow 0
\end{aligned}
$$

To prove $f(x, y, z)=0$ with the method of [Kounalis and Rusinowitch, 1990a], we instanciate $x, y$ and $z$ by 0 and $s\left(x^{\prime}\right)$ (from $S(R)$ ) in all possible ways. We obtain 8 equations and the proof of some of them diverges. With the method presented here, thanks to case rewriting, we do not need to consider all these inductive positions. We have: $O c c \_i n d(p)=\{2\}$ and $O c c \_i n d(f)=\emptyset$. To prove $f(x, y, z)=0$, we apply case rewriting to get $p(y, x, z)=$ True $\Rightarrow 0=0$ and $p(y, x, z)=$ True. The first clause is a tautology and the second one is proved by instanciating $x$ by 0 and $s(x)$.

Other authors have applied case rewriting techniques for inductive theorem proving. Among them [Bronsard and Reddy, 1990] and [Bevers, 1993] propose an approach related to ours but their methods cannot be considered as automatic, since they cannot check the applicability of case rewriting rules due to the fact that their provers are restricted to Horn clauses.

To conclude, our new case rewriting rule avoids many drawbacks of the previously defined ones and it allows to prove a larger class of theorems.

\section{A Proof Procedure for Conditional Theories}

\subsection{Inferences rules}

We present our procedure for proof by induction as a set of inference rules to be applied fairly to the goals. Let $R$ be a rewrite system for the set of axioms $A x$, we suppose that any defined function is completely defined. The procedure modifies incrementally two sets of clauses $E$ and $H$, where $E$ contains the conjectures to be checked and $H$ contains clauses, previously in $E$, that have been reduced and can therefore be used as inductive hypotheses. This procedure is refutational in essence, and performs implicit induction w.r.t. to $\succ$. Its correctness is obtained by very simple arguments about the existence of a minimal counterexample. We think that our correctness proof is much simpler than the related ones [Reddy, 1990]. As a consequence it 


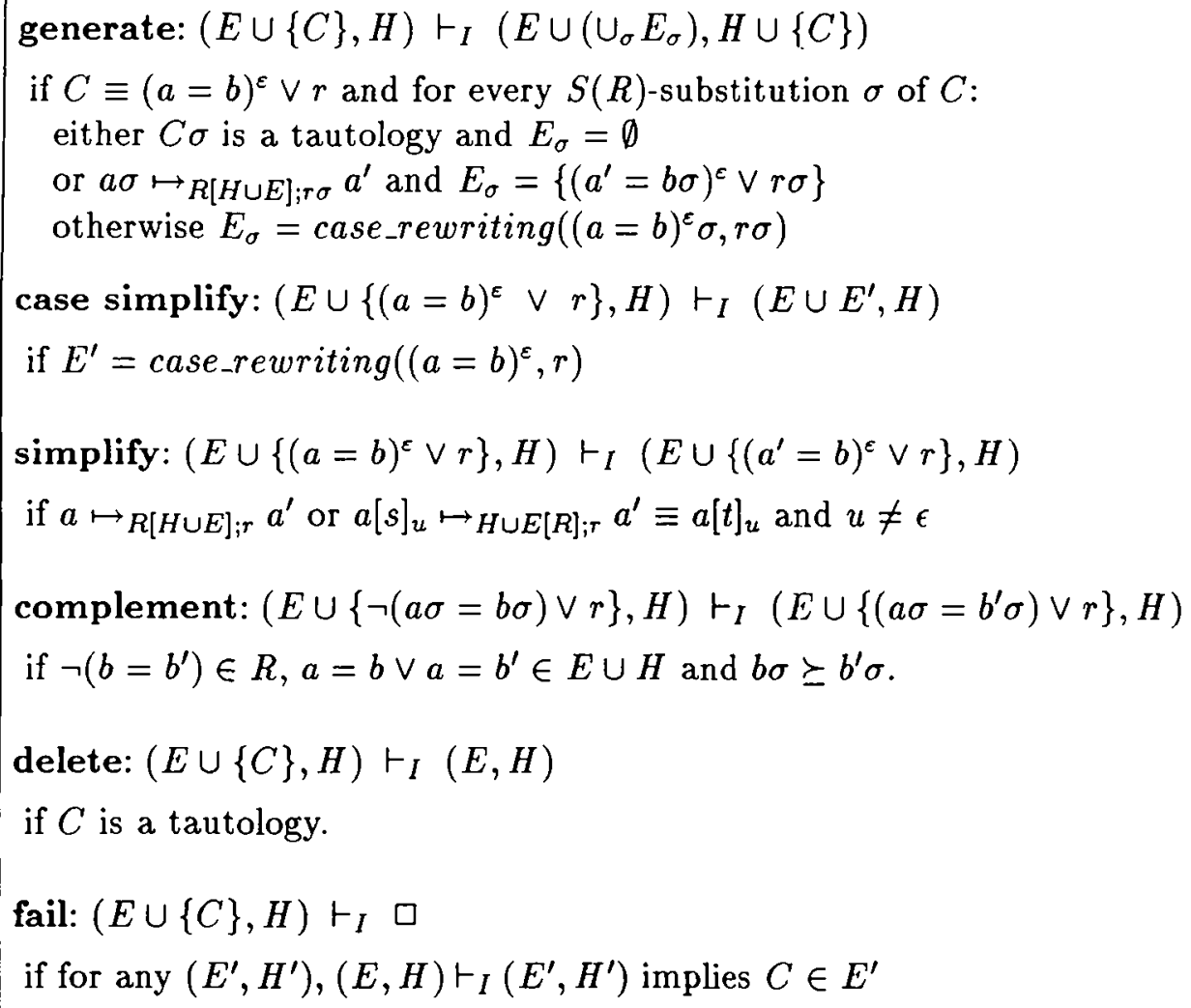

Figure 1: Inference System $I$

was easy for us to add many optimizations to the procedure and show that they do not affect correctness. The inference system for induction $I$ contains the rules given in figure 1 .

The generate rule allows to derive lemmas and initiates induction steps. The case simplify rule simplifies a conjecture with conditional rules and adds to the result the contexts where the respective reductions are valid. The simplify rule reduces a clause $C$ with axioms from $R$, induction hypotheses from $H$ and other conjectures (therefore we can simulate simultaneous induction). The premisses of $C$ considered as a conditional axiom can also help to check that the preconditions of a rule being applied to $C$ are valid. The complement rule transforms negative clauses to positive clauses that are easier to refute. The role of deletion is obvious. The fail rule is applied to $(E, H)$ if no other rule can be applied to $C \in E$.

An $I$-derivation is a sequence of states:

$$
\left(E_{0}, \emptyset\right) \vdash_{I}\left(E_{1}, H_{1}\right) \vdash_{I} \ldots \vdash_{I}\left(E_{n}, H_{n}\right) \vdash_{I} \ldots
$$

An $I$-derivation fails if it terminates with the rule fail.

In our former procedure of [Bouhoula et al., 1992a] the generate rule was conditionned by ordering restrictions. The term $a$ was bound to be a maximal member of an equation (w.r.t. $\succ$ ). The same restriction is valid in the actual setting and was omitted for clarity of presentation. Moreover the induction variables that have to be considered for building $S(R)$-substitutions can be selected out from maximal members of literals of the clauses. These additional constraints are crucial for avoiding divergence in many cases. 


\subsection{Correctness of the procedure}

The correctness of $I$ is obtained by defining a well-founded ordering on clauses and a notion of fair derivation. Fairness roughly means that every clause in the set of conjectures will be eventually modified by some inference.

Then we reason by contradiction: if a non valid clause is generated in a non failed derivation then a minimal one is generated too. We show that no inference step can apply to this clause. In other words, this clause persists in the derivation. This is a contradiction with the fairness hypothesis. The well-founded ordering on clauses is defined by first introducing the complexity of an equation. The complexity of an equation $g=h$ is defined as:

$$
C(g=h)=\left\{\begin{array}{l}
(\{g\},\{h\}) \text { if } g \succ h \\
(\{h\},\{g\}) \text { if } g \prec h \\
(\{g, h\}, \perp) \text { otherwise }
\end{array}\right.
$$

where the new symbol $\perp$ is taken to be minimal in $\ll$. We define an ordering on equations as follows: $(a=b) \prec_{e}(c=d)$ iff $C(a=b)$ is smaller than $C(c=d)$ for the lexicographic composition of $\ll$ on the first and second components of the complexity. The multiset extension of $\prec_{e}$ will be denoted by $\varkappa_{e}$.

Let $C$ be a clause of type $\wedge_{i} a_{i}=b_{i} \Rightarrow \vee_{j} c_{j}=d_{j}$. We define $\operatorname{Rep}(C)=\left\{C\left(a_{i}=b_{i}\right)\right\}_{i} \cup\left\{C\left(c_{j}=\right.\right.$ $\left.\left.d_{j}\right)\right\}_{j}$. Given two clauses $C_{1}, C_{2}$, we say that $C_{1} \prec_{c} C_{2}$ if lexicographically $\operatorname{Rep}\left(C_{1}\right) \varkappa_{e} \operatorname{Rep}\left(C_{2}\right)$ or $n \ln \left(C_{1}\right)<n \ln \left(C_{2}\right)$, where $n \ln (C)$ is the number of negative literals of $C$.

Example 6.1 Let $C_{1} \equiv \neg(c=d) \vee a=b$ and $C_{2} \equiv a^{\prime}=b \vee c^{\prime}=d^{\prime}$ with $a * b, a * a^{\prime}, a^{\prime} \prec$ $b, c \succ d, c^{\prime} \succ d^{\prime}$ and $c^{\prime} \prec c$ then $C_{2} \prec_{c} C_{1}$.

The correctness of a procedure based on our inference system relies on a fairness assumption: every conjecture to be checked must be considered at some step. More formally, a derivation $\left(E_{0}, H_{0}\right) \vdash_{I}\left(E_{1}, H_{1}\right) \vdash_{I} \ldots$ is fair if either it fails or it is infinite and the set of persisting clauses $\left(\cup_{i \geq 0} \cap_{j \geq i} E_{j}\right)$ is empty.

Lemma 2 Let $\left(E_{0}, \emptyset\right) \vdash_{I}\left(E_{1}, H_{1}\right) \vdash_{I} \ldots$ be a fair I-derivation such that $R \nvdash_{\text {ind }} E_{0}$ then there exists $i$ such that the rule fail applies to $\left(E_{i}, H_{i}\right)$.

proof: Let $C \theta$ be a minimal element w.r.t. $\prec_{c}$ of the set $\left\{D \sigma / D \in \cup_{i} E_{i}\right.$ and there is a ground $R$-irreducible substitution $\sigma$ such that $\left.R \nvdash_{\text {ind }} D \sigma\right\}$. $C$ exists since $R \nvdash_{\text {ind }} E_{0}$ and $\prec_{c}$ is wellfounded. Assume that $C \in E_{j}$. Then there exists $k \geq j$ such that the rule fail applies to $\left(E_{k}, H_{k}\right)$. It is sufficient to check that $C$ cannot be simplified nor deleted, and that neither generate nor complement apply to $C$. As a consequence fail applies since the clause $C$ must not persist in the derivation by the fairness hypothesis. The detailed proof is found in Appendix 2.

The next theorem is a straightforward consequence of the above lemma:

Theorem 6.1 (correctness) Let $R$ be a rewrite system and let $\left(E_{0}, \emptyset\right) \vdash_{I}\left(E_{1}, H_{1}\right) \vdash_{I} \ldots$ be a fair I-derivation. If it does not fail then $R \models_{\text {ind }} E_{0}$.

Since every I-derivation from $(E, \emptyset)$ to $(\emptyset, H)$, where $H$ is some set of clauses, is fair then the conjectures of $E$ are inductive consequences of $R$. This remark is important from a practical point of view. Note also that $E_{0}$ is valid even when the derivation is infinite. 


\subsection{Refutation of conjectures}

From now on, we assume that $R$ is a ground convergent rewrite system such that all its defined symbols are completely defined. Moreover, if the defined function $g$ appears in a l.h.s. of a conditional rule, then every rule that contains $g$ in its l.h.s. is linear. Finally, we assume that every left-hand side of a conditional rule has a defined symbol. Let us call $J$ the set of inference rules obtained by adding to $I$ the rule:

disproof: $(E \cup\{C\}, H) \vdash_{J}$ Disproof if $C$ is quasi-inconsistent.

A J-derivation fails if it ends with fail. If disproof is applied then a quasi-inconsistent clause is detected and therefore, from theorem 4.1, we can conclude that some conjecture is false:

Corollary 1 Let $\left(E_{0}, \emptyset\right) \vdash_{J}\left(E_{1}, H_{1}\right) \vdash_{J} \ldots$ be a J-derivation. If there is a $k$ such that disproof applies to $\left(E_{k}, H_{k}\right)$ then $R \forall_{\text {ind }} E_{k}$.

If at step $k$, we find that $R \forall_{\text {ind }} E_{k}$, we can conclude that $E_{0}$ is not valid either in $R$. This is a consequence of the next result:

Lemma 3 Let $\left(E_{0}, \emptyset\right) \vdash_{J}\left(E_{1}, H_{1}\right) \vdash_{J} \ldots$ be a J-derivation. If for all $j$ such that $j<i$ we have $R \models_{\text {ind }} E_{j}$ then $R \models_{\text {ind }} E_{i}$.

proof: If $\left(E_{k-1}, H_{k-1}\right) \vdash_{J}\left(E_{k}, H_{k}\right)$ by generate on $C$, consider $C \sigma$ which is an instance of $C$ by a $S(R)$-substitution. If $C \sigma$ is simplified then every auxiliary equation which is used for rewriting is either in $R$ or $E_{j^{\prime}}\left(j^{\prime}<k\right)$ and hence $E_{k}$ is valid in $R$. If case_rewriting is applied to $C \sigma$ then $E_{k}$ is valid in $R$ since all defined symbols are completely defined, by hypothesis. For case simplify the argument is the same as above.

If $\left(E_{k-1}, H_{k-1}\right) \vdash_{J}\left(E_{k}, H_{k}\right)$ by simplify then the equations which are used for simplification occur in some $E_{j}(j<k)$ and therefore are valid in $R$ by hypothesis. Hence, $E_{k}$ is valid too in $R$.

If $\left(E_{k-1}, H_{k-1}\right) \vdash_{J}\left(E_{k}, H_{k}\right)$ by complement. Suppose that complement is applied to $C \equiv$ $\neg(a \theta=b \theta) \vee r$ to obtain $C^{\prime} \equiv\left(a \theta=b^{\prime} \theta\right) \vee r$. We can show that $R \models_{\text {ind }} C^{\prime}$. The detailed proof is found in Appendix 2.

Theorem 6.2 Let $R$ be a ground convergent rewrite system such that every defined symbol is completely defined and let $\left(E_{0}, \emptyset\right) \vdash_{J}\left(E_{1}, H_{1}\right) \vdash_{J} \ldots$ be a $J$-derivation. If there exists $j$ such that disproof applies to $\left(E_{j}, H_{j}\right)$ then $R \not \models E_{0}$.

proof: Let $\left(E_{0}, \emptyset\right) \vdash_{J}\left(E_{1}, H_{1}\right) \vdash_{J} \ldots$ be a J-derivation. Assume that there is $j$ such that disproof applies to $\left(E_{j}, H_{j}\right)$. It is clear from corollary 1 that $R \forall_{i n d} E_{j}$ and by lemma 3 , we have $R \not \forall_{\text {ind }} E_{0}$.

\section{A refutationally complete procedure for theories with boolean preconditions}

In this section we shall consider axioms that are conditional rules with boolean preconditions over free constructors. To be more specific, we assume there exists a sort bool with two nullary free constructors $\{$ True, False $\}$. Every rule in $R$ is of type: $\wedge_{i=1}^{n} p_{i}=p_{i}^{\prime} \Rightarrow s \rightarrow t$ where for all $i$ in $[1 \ldots n], p_{i}^{\prime} \in\{$ True, False $\}$. Such a system $R$ is called a boolean rewrite system. For $\alpha \in\{$ True, False $\}$ we denote by $\alpha^{-}$the complementary bool symbol of $\alpha$. Conjectures 
will be boolean clauses, that is clauses whose negative literals are of type $\neg\left(p=p^{\prime}\right)$ where $p^{\prime} \in\{$ True, False $\}$.

We also assume that any function symbol $p$ with boolean values is completely defined. In other words, the following is inductively valid:

$$
p(\vec{x})=\text { True } \vee p(\vec{x})=\text { False }
$$

Therefore, the following propositions are also valid and they can be used to eliminate negations:

$$
\neg(p(\vec{x})=\text { True }) \Leftrightarrow(p(\vec{x})=\text { False }), \neg(p(\vec{x})=\text { False }) \Leftrightarrow(p(\vec{x})=\text { True })
$$

We can then define a new inference system $K$ from $I$ by reformulating complement as follows:

complement: $(E \cup\{\neg(a=\alpha) \vee r\}, H) \vdash_{K}\left(E \cup\left\{\left(a=\alpha^{-}\right) \vee r\right\}, H\right)$ if $\alpha \in\{$ True, False $\}$.

and replacing the fail rule by:

disproof: $(E \cup\{C\}, H) \vdash_{K}$ Disproof if for any $\left(E^{\prime}, H^{\prime}\right),(E, H) \vdash_{K}\left(E^{\prime}, H^{\prime}\right)$ implies $C \in E^{\prime}$.

A K-derivation fails if it ends with disproof. The inference system $K$ allows to refute false conjectures, thanks to the following results:

Lemma 4 Let $C \equiv(a=b)^{\varepsilon} \vee r$. If a contains a defined symbol then generate can be applied to $(E \cup\{C\}, H)$.

proof: Let $C \equiv(a=b)^{\varepsilon} \vee r$ and let $\sigma$ be an $S(R)$-substitution of $C$. The term $a$ contains a term $s$ of the form $f\left(t_{1}, \ldots, t_{n}\right)$ where $f$ is a defined symbol and for all $i, t_{i}$ is in $T(C, X)$. The term $s \sigma$ matches a left-hand side of $R$, otherwise there exists a substitution $\tau$ such that $s \sigma \tau$ is ground and strongly irreducible by $R$, by using clause b. of proposition 4.1 . This leads to a contradiction since we have assumed that $f$ is completely defined. On the other hand, $\sigma$ is an $S(R)$-substitution of $C$, therefore $s \sigma$ does not contain an inductive variable. So either some inductive rewriting or some case rewriting can be applied to $C \sigma$ and therefore generate can be applied to $C$. This ends the proof of lemma 4 .

If disproof is applied in a $K$-derivation, then there exists a boolean clause $C$ such that generate cannot be applied to $C$. Therefore there exists a $S(R)$-substitution $\sigma$ such that $C \sigma$ is not a tautology. Moreover by lemma 4,C $\sigma$ cannot contain a defined symbol. Hence it only contains constructor symbols and therefore, since constructors are free by hypothesis, it is strongly $R$ irreducible. As a consequence, $C$ is a quasi-inconsistent clause.

Note now that the only rule that permits to introduce negative clauses is case_rewriting. Let us assume that $E_{0}$ only contains boolean clauses. Since the axioms have boolean preconditions, all the clauses generated in a $K$-derivation are boolean. So the new inference system $K$ can be proved refutationally complete for boolean clauses as well.

Theorem 7.1 Let $R$ be a ground convergent boolean rewrite system such that every defined symbol is completely defined and let $\left(E_{0}, \emptyset\right) \vdash_{K}\left(E_{1}, H_{1}\right) \vdash_{K} \ldots$ be a fair $K$-derivation such that $E_{0}$ only contains boolean clauses. Then $R \not \forall_{i n d} E_{0}$ iff the derivation fails. 


\section{Optimizations}

In this section, we shall enhance our inference system $J$ by new simplification rules to handle non orientable equations and gain efficiency. In particular, we allow under some conditions a term $s$ to be rewritten to another one that is not comparable with $s$. For this purpose we define a new rewrite relation that we call relaxed inductive rewriting:

Definition 8.1 (relaxed inductive rewriting) Let $R$ and $W$ be two sets of conditional equations. Consider a clause $C \equiv(a=b)^{\varepsilon} \vee r$ and its skolemized version $\bar{C} \equiv(\bar{a}=\bar{b})^{\varepsilon} \vee \bar{r}$. We write: $a \sim R[W] ; r$ a $a^{\prime}$ if:

either $\bar{a} \rightarrow_{\operatorname{prem}(\bar{r})} \overline{a^{\prime}}$ and $a \succ a^{\prime}$,

or there exists a position $u$ in $a$, a substitution $\sigma$ and a conditional equation $\wedge_{i=1}^{n} a_{i}=b_{i} \Rightarrow s=t$ in $R$ such that:

1. $a \equiv a[s \sigma]_{u}$ and $a^{\prime} \equiv a[t \sigma]_{u}$.

2. $\left\{a[s \sigma]_{u}\right\} \gg\left\{a_{1} \sigma, b_{1} \sigma, \ldots, a_{n} \sigma, b_{n} \sigma\right\}$.

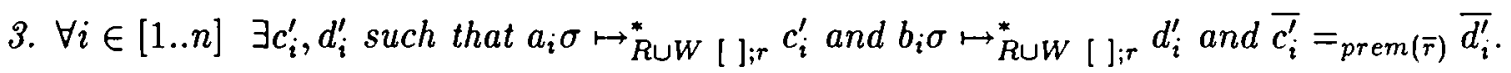

Note that lemma 1 remains valid if we replace $\mapsto_{R[W] ; r}$ by $\sim R[W]_{;}$.

Consider now the inference $N$ obtained by adding to $J$ the following rules:

right simplify of constructors: $(E \cup\{f(\vec{s})=f(\vec{t}) \vee r\}, H) \quad \vdash_{N}\left(E \cup\left(\cup_{i}\left\{s_{i}=t_{i} \vee r\right\}_{i}\right), H\right)$

if $f$ is a free constructor.

left simplify of constructors: $(E \cup\{\neg f(\vec{s})=f(\vec{t}) \vee r\}, H) \quad \vdash_{N}\left(E \cup\left\{\vee_{i} \neg\left(s_{i}=t_{i}\right) \vee r\right\}, H\right)$ if $f$ is a free constructor.

subsumption: $(E \cup\{C\}, H) \vdash_{N}(E, H)$

if $C$ is subsumed by another clause of $R \cup H \cup E$.

and replacing the simplify rule by:

$$
\begin{aligned}
& \text { simplify: }\left(E \cup\left\{(a=b)^{\varepsilon} \vee r\right\}, H\right) \vdash_{N}\left(E \cup\left\{\left(a^{\prime}=b\right)^{\varepsilon} \vee r\right\}, H\right) \\
& \text { if } a \leadsto R[H \cup E]_{; r} a^{\prime} \text { or } a[s]_{u} \mapsto H \cup E[R]_{; r} a^{\prime} \equiv a[t]_{u}, u \neq \epsilon \text { and } a^{\prime}=b \prec_{e} a=b .
\end{aligned}
$$

Simplify makes possible to simplify non orientable equations derived in $H$ or $E$, such as commutativity, when standard inductive rewriting fails. Left simplification of constructors and right simplification of constructors take advantage that constructors are free to decompose terms. Subsumption delete clauses $C$ subsumed by an element of $R$ or $H \cup(E \backslash\{C\})$.

Note that lemma 2 remains true when we replace I-derivations by $\mathrm{N}$-derivations. Therefore theorem 6.1 is valid and the inference system $N$ is correct. Refutational completeness is also preserved for boolean systems. The detailed proof is found in Appendix 2. 


\section{Computer Experiments}

Our prototype SPIKE (written in Caml Light) is designed to prove the validity of a set of clauses in a conditional theory. The first step in a proof session is to check if all defined functions are completely defined. If this step is successful then we can use a more efficient version of the case rewriting rule. The second step is to check the ground convergence of the set of axioms. If the first two steps are successful then we can refute false conjectures. The third step is to compute test sets and inductive positions. After these preliminary tasks, the proof starts.

Example 9.1 Consider example 2.1 and let us prove the conjecture sorted $(\operatorname{insert}(x 1, x 2))=$ sorted $(x 2)$. Note that $R R L$ [Zhang et al., 1988] is unable to succeed with this example unless the user suggests some well-chosen lemmas.

All functions are completely defined;

$R$ is ground convergent;

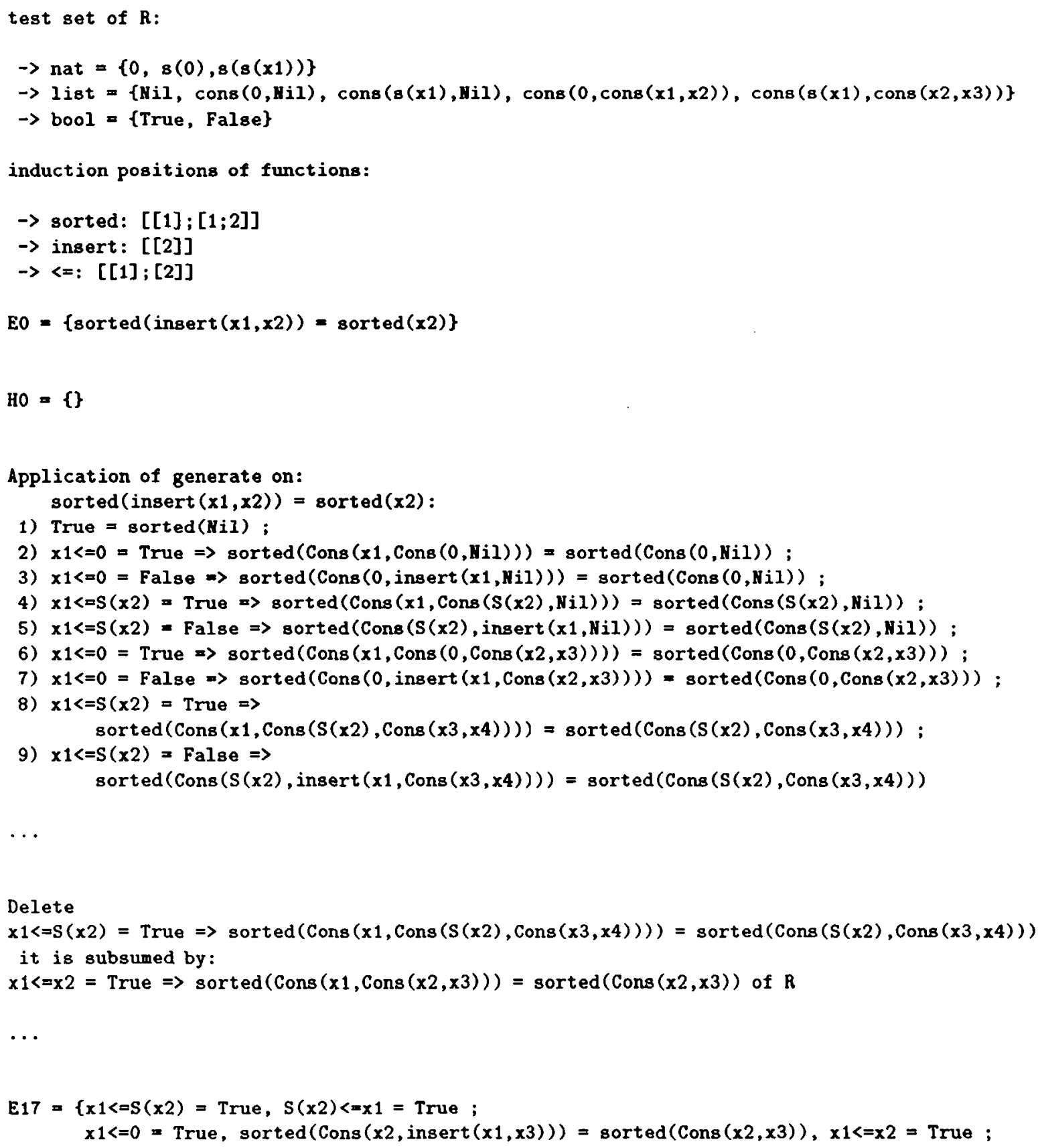




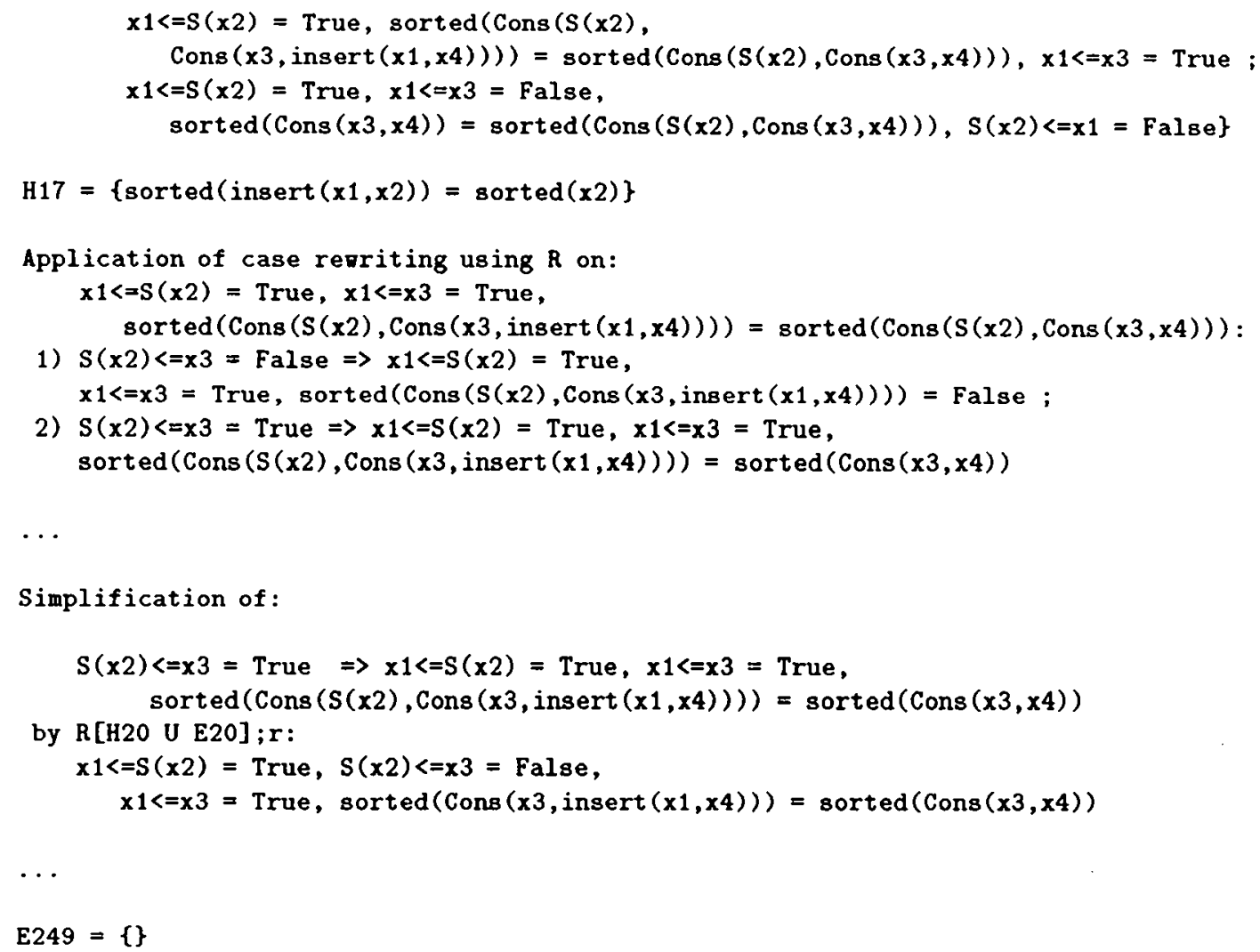

The initial conjectures are inductive consequences of $R$

The following sub lemmas have been generated automatically during the proof and have played a role in it:

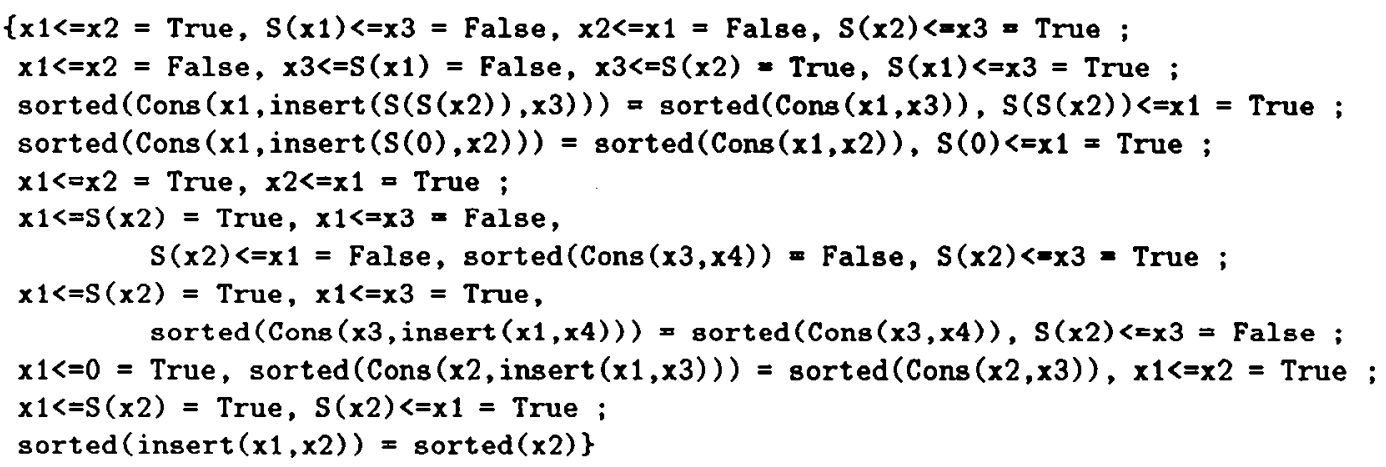

\section{Conclusion}

We have proposed a new procedure for proof by induction in conditional theories. Our procedure relies on the implicit induction paradigm and puts the stress on simplification and case analysis. As our previous procedure [Bouhoula et al., 1992a], it allows simplification of conjectures by conjectures and has been extended to handle non-orientable equations. It can also refute non valid conjectures. A main contribution of this paper is that our strategy is refutationally complete for a class of rewrite systems that can specify numerous interesting examples. This class contains the boolean ground convergent rewrite systems with completely defined functions over free constructors. In other words with our procedure every false conjecture will be disproved in finite time. However, our method remains valid even when the functions are not completely defined. Note that our correctness and completeness proofs do not require an elaborated notion of fairness. 
We plan to enhance the system with generalisation techniques for suggesting lemmas, as the one proposed in [Basin and Walsh, 1993], since for many examples the "Generate" rule is not sufficient for deriving the lemmas needed for achieving a goal. Also extension of the method to parametrized specifications should lead to shorter and structured proofs. The format of implicit induction is not user-friendly. Therefore, some effort should be devoted to understand the relationship between our method and explicit induction (in particular, in the case of mutual induction [Walther, 1993]). We shall explore the possibility of translations between the two frameworks. If such a translation is available then we should benefit from recent promising works on proof planning [Ireland, 1992].

An extension to theories that are presented by stratified sets of clauses should also follow easily from our work.

Acknowledgements: We thank Emmanuel Kounalis, Hélène Kirchner and Maria Huber for their valuable comments.

\section{References}

[Bachmair, 1988] L. Bachmair. Proof by consistency in equational theories. In Proceedings 3rd IEEE Symposium on Logic in Computer Science, Cambridge (Mass., USA), pages 228-233, 1988.

[Basin and Walsh, 1993] D. Basin and T. Walsh Difference Unification. In Proceedings of 13th IJCAI, Morgan Kaufmann Publishers, volume 1, pages 116-122, 1993

[Bevers, 1993] E. Bevers. Automated Reasoning in Conditional Algebric Specifications: Termination and Proof by Consistency. PhD thesis, Katholieke Universiteit Leuven, Belgium, 1993.

[Bouhoula et al., 1992a] A. Bouhoula, E. Kounalis, and M. Rusinowitch. Automated mathematical induction. Technical Report 1636, INRIA, 1992.

[Bouhoula et al., 1992b] A. Bouhoula, E. Kounalis, and M. Rusinowitch. Spike: an automatic theorem prover. In Proceedings of LPAR'92, volume 624 of LNAI, Saint Petersbourg, Russia, July 1992. Springer-Verlag.

[Boyer and Moore, 1979] R. S. Boyer and J. S. Moore. A Computational Logic. Academic Press, New York, 1979.

[Bronsard and Reddy, 1990] F. Bronsard and S. Reddy. Conditional rewriting in focus. In S. Kaplan and M. Okada, editors, Proceedings of the 2nd Workshop on Conditional and Typed Rewriting Systems, volume 516 of LNCS. Springer-Verlag, 1990.

[Bundy et al., 1989] A. Bundy, F. van Harmelen, A. Smaill, and A. Ireland. Extensions to the rippling-out tactic for guiding inductive proofs. In M. E. Stickel, editor, 10th International Conference on Automated Deduction, volume 449 of LNAI, pages 132-146. Springer-Verlag, July 1989.

[Chadha and Plaisted, 1992] R. Chadha and D.A. Plaisted. Mechanizing mathematical induction. Presented at the Second International Symposium on Artificial Intelligence and Mathematics, Fort Lauderdale, Florida, 1992.

[Dershowitz and Jouannaud, 1990] N. Dershowitz and J.-P. Jouannaud. Rewrite systems. In J. van Leuven, editor, Handbook of Theoretical Computer Science. Elsevier Science Publishers North-Holland, 1990. 
[Dershowitz et al., 1988] N. Dershowitz, M. Okada, and G. Sivakumar. Canonical conditional rewrite systems. In Proceedings 9th International Conference on Automated Deduction, Argonne (Ill., USA), volume 310 of LNCS. Springer-Verlag, May 1988.

[Fribourg, 1986] L. Fribourg. A strong restriction of the inductive completion procedure. In Proceedings 13th International Colloquium on Automata, Languages and Programming, volume 226 of $L N C S$, pages 105-115. Springer-Verlag, 1986.

[Guttag, 1978] J. Guttag. Abstract data types and software validation. Communications of the $A C M$, volume 21, pages 1048-1064, 1978.

[Huber, 1991] M. Huber. Test-set approaches for ground reducibility in term rewriting systems, characterizations and new applications. Master's thesis, Technische Universität Berlin, 1991.

[Huet and Hullot, 1982] G. Huet and J.-M. Hullot. Proofs by induction in equational theories with constructors. Journal of Computer and System Sciences, 25(2):239-266, October 1982.

[Huet, 1991] G. Huet. The Gilbreath trick: a case study in axiomatisation and proof development in the coq proof assistant. Technical Report 1511, INRIA, 1991.

[Ireland, 1992] A. Ireland. The use of planning critics in mechanizing inductive proofs. In Proceedings of the International Conference on Logic Programming and Automated Reasoning, volume 624 of LNAI. Springer-Verlag, 1992.

[Jouannaud and Kounalis, 1986] J.-P. Jouannaud and E. Kounalis. Proof by induction in equational theories without constructors. In Proceedings 1st IEEE Symposium on Logic in Computer Science, Cambridge (Mass., USA), pages 358-366, 1986.

[Kapur and Musser, 1987] D. Kapur and D. Musser. Proof by consistency Artificial Intelligence, volume $31(2)$, pages $125-157,1987$

[Kounalis and Rusinowitch, 1990a] E. Kounalis and M. Rusinowitch. A mechanization of conditional reasoning. In First International Symposium on Artificial Intelligence and Mathematics, Fort Lauderdale, Florida, January 1990.

[Kounalis and Rusinowitch, 1990b] E. Kounalis and M. Rusinowitch. Mechanizing inductive reasoning. In Proceedings of the American Association for Artificial Intelligence Conference, Boston, pages 240-245. AAAI Press and MIT Press, July 1990.

[Kounalis, 1990] E. Kounalis. Testing for inductive (co)-reducibility. In A. Arnold, editor, Proceedings 15th CAAP, Copenhagen (Denmark), volume 431 of LNCS, pages 221-238. Springer-Verlag, May 1990.

[Musser, 1980] D. R. Musser. On proving inductive properties of abstract data types. In Proceedings 7th ACM Symp. on Principles of Programming Languages, pages 154-162. Association for Computing Machinery, 1980.

[Padawitz, 1988] P. Padawitz. Computing in Horn Clause Theories, Springer Verlag, 1988.

[Reddy, 1990] U. S. Reddy. Term rewriting induction. In M. E. Stickel, editor, Proceedings 10th International Conference on Automated Deduction, Kaiserslautern (Germany), volume 449 of $L N C S$, pages 162-177. Springer-Verlag, 1990.

[Walther, 1993] C. Walther. Combining Induction Axioms By Machine. In Proceedings of 13th IJCAI, Morgan Kaufmann Publishers, volume 1, pages 95-100, 1993 
[Zhang et al., 1988] H. Zhang, D. Kapur, and M. S. Krishnamoorthy. A mechanizable induction principle for equational specifications. In E. Lusk and R. Overbeek, editors, Proceedings 9th International Conference on Automated Deduction, Argonne (Ill., USA), volume 310 of LNCS, pages 162-181. Springer-Verlag, 1988.

[Zhang, 1993] H. Zhang Implementing Contextual Rewriting Proceedings of the 3rd CTRS Workshop, volume 656 of $L N C S$. Springer-Verlag, 1993. 


\section{Appendix 1: The Gilbreath Card Trick}

\section{A Introduction}

Suppose you have a deck of cards of even length. Suppose the cards alternate between red ones and black ones. Cut the deck into two piles, $a$ and $b$. Shuffle $a$ and $b$ together. Then the following is true of the shuffled deck. If the bottom-most cards in $a$ and $b$ are of different color, then when the cards of the shuffled deck are taken from the top in adjacent pairs, each pair contains a card of each color. On the other hand, if the bottom-most cards in a and b are the same color, the above pairing property holds after rotating the shuffled deck by one card, i.e., moving the bottom card to the top.

An interactive proof of Gilbreath Cards Trick was first given by G. Huet [Huet, 1991] using the COQ proof assistant. B. Boyer has used NQTHM to derive another proof. A similar but much faster proof was obtained by $\mathrm{H}$. Zhang with RRL. These two automatized proofs require many lemmas, some of them being non-obvious. For instance, B. Boyer introduces a predicate "silly" that is "only defined to force a certain weird induction" (here we quote B. Boyer). The same predicate appears in Zhang's experiment. On the other hand, our proof is based on 5 lemmas easy to understand. These lemmas have been suggested by a first unsuccessful proof attempt with SPIKE. The source of failure was identified by the impossibility of reducing a family of patterns. Hence we have introduced the adequate lemmas for simplifying them. This was enough to derive a proof. Note that if we use the same formulation as H. Zhang, our system needs only 2 lemmas to prove the Gilbreath Cards Trick (see section D).

The proof has taken more CPU time than Boyer's (and Zhang's). However, it is difficult to compare these approaches from the efficiency point of view, since our we have spent very few time to get the right lemmas. On the other hand, the differences between programming languages lead also to some discrepancy in the performance.

The following array compare users inputs for the proof of Gilbreath Card Trick with NQTHM, RRL and SPIKE:

\begin{tabular}{|l|l|l|l|l|l|}
\hline & NQTHM & \multicolumn{2}{|c|}{ RRL } & \multicolumn{2}{|c|}{ SPIKE } \\
\hline Definitions & $?$ & 10 & 10 & 9 & 10 \\
\hline Induction scheme definition & 1 & 1 & 0 & 0 & 0 \\
\hline Lemmas & 17 & 23 & 8 & 5 & 2 \\
\hline
\end{tabular}

In chronological order we have: NQTHM, first column of RRL, first column of SPIKE, second column of RRL, corresponding, to more recent experiments obtained after modification of the code of RRL, second column of SPIKE corresponding to same inputs as RRL.

\section{B Formulation of the problem in SPIKE}

In this section, we define in SPIKE various predicates and functions to formulate the Gilbreath Card Trick:

There are two kind of cards: $R=$ red and $B=$ black.

$$
\begin{aligned}
& R: \rightarrow \text { card } \\
& B: \rightarrow \text { card }
\end{aligned}
$$

The constructors for lists of cards:

$$
\begin{aligned}
& N u l l: \rightarrow \text { list } \\
& \text { Cons }: \text { card } \times \text { list } \rightarrow \text { list }
\end{aligned}
$$

The constructors for boolean: 
True $: \rightarrow$ bool

False : $\rightarrow$ bool

We suppose that True $<>$ False:

True $=$ False $\Rightarrow$

$[$ neg $:$ card $\rightarrow$ card $]$ The negation function maps $\mathrm{R}$ to $\mathrm{B}$ and conversely:

$n e g(R)=B$

$n e g(B)=R$

[paired $:$ card $\times$ card $\rightarrow$ bool $]$ paired $(x, y)=$ true iff $x$ and $y$ are cards of opposite color.

paired $(R, B)=$ True

paired $(B, R)=$ True

paired $(x, x)=$ False

[append $:$ list $\times$ list $\rightarrow$ list $]$ appends two lists.

append $(N u l l, y)=y$

$\operatorname{append}(\operatorname{Cons}(x, y), z)=\operatorname{Cons}(x, \operatorname{append}(y, z))$

$[$ rotate $:$ list $\rightarrow$ list $]$ rotate the first element to the end of a list.

$\operatorname{rotate}(N u l l)=N u l l$

$\operatorname{rotate}(\operatorname{Cons}(x, y))=\operatorname{append}(y, \operatorname{Cons}(x, N u l l))$

[even : list $\rightarrow$ bool $]$ even $(x)=$ true iff $x$ is of even length.

$\operatorname{even}(N u l l)=$ True

even $(\operatorname{Cons}(x, N$ ull $))=$ False

even $(\operatorname{Cons}(x 1, \operatorname{Cons}(x 2, y)))=\operatorname{even}(y)$

[opposite $:$ list $\times$ list $\rightarrow$ bool] opposite $(x, y)=$ true iff the first cards of $x$ and $y$, respectively, are of opposite color.

opposite $(N u l l, y)=$ False

opposite $(x, N$ ull $)=$ False

opposite $(\operatorname{Cons}(x 1, y 1), \operatorname{Cons}(x 2, y 2))=\operatorname{paired}(x 1, x 2)$

[pairedlist : list $\rightarrow$ bool] pairedlist $(x)=$ true iff $x$ is a list of cards such that if we repeatedly take two cards from its top, the two cards are found to be of opposite color.

pairedlist $(N u l l)=$ True

pairedlist $(\operatorname{Cons}(x, N$ ull $))=$ True

paired $(x 1, x 2)=$ True $\Rightarrow$ pairedlist $(\operatorname{Cons}(x 1, \operatorname{Cons}(x 2, x)))=$ pairedlist $(x)$

paired $(x 1, x 2)=$ False $\Rightarrow$ pairedlist $(\operatorname{Cons}(x 1, \operatorname{Cons}(x 2, x)))=$ False

[alter $:$ list $\rightarrow$ bool $]$ alter $(x)=$ true iff $x$ is a list of cards whose colors alternate.

$\operatorname{alter}(N u l l)=$ True

alter $(\operatorname{Cons}(x 1, N u l l))=$ True

paired $(x 1, x 2)=\operatorname{True} \Rightarrow \operatorname{alter}(\operatorname{Cons}(x 1, \operatorname{Cons}(x 2, x)))=\operatorname{alter}(\operatorname{Cons}(x 2, x))$

paired $(x 1, x 2)=$ False $\Rightarrow$ alter $(\operatorname{Cons}(x 1, \operatorname{Cons}(x 2, x)))=$ False

[shuffle: list $\times$ list $\times$ list $\rightarrow$ bool $]$ shuffle $(x, y, z)=$ true iff $z$ is a merge of $x$ and $y$.

shuffle $(N$ ull, $N$ ull, $N$ ull $)=$ True

$\% \% \operatorname{shuffle}(x, N u l l, z) \Leftrightarrow(x=z)$

shuffle $(N$ ull, $N$ ull, Cons $(x 3, y 3))=$ False

shuffle(Cons $(x 1, y 1), y 2, N u l l)=$ False

paired $(x 1, x 3)=$ True $\Rightarrow$ shuffle $(\operatorname{Cons}(x 1, y 1), N$ ull, Cons $(x 3, y 3))=$ False

paired $(x 1, x 3)=$ False $\Rightarrow$ shuffle $(\operatorname{Cons}(x 1, y 1), N$ ull, Cons $(x 3, y 3))=\operatorname{shuffle}(y 1, N u l l, y 3)$

$\% \%$ shuffle $(N u l l, x, z) \Leftrightarrow(x=z)$

shuffle(y1, Cons $(x 2, y 2), N u l l)=$ False 


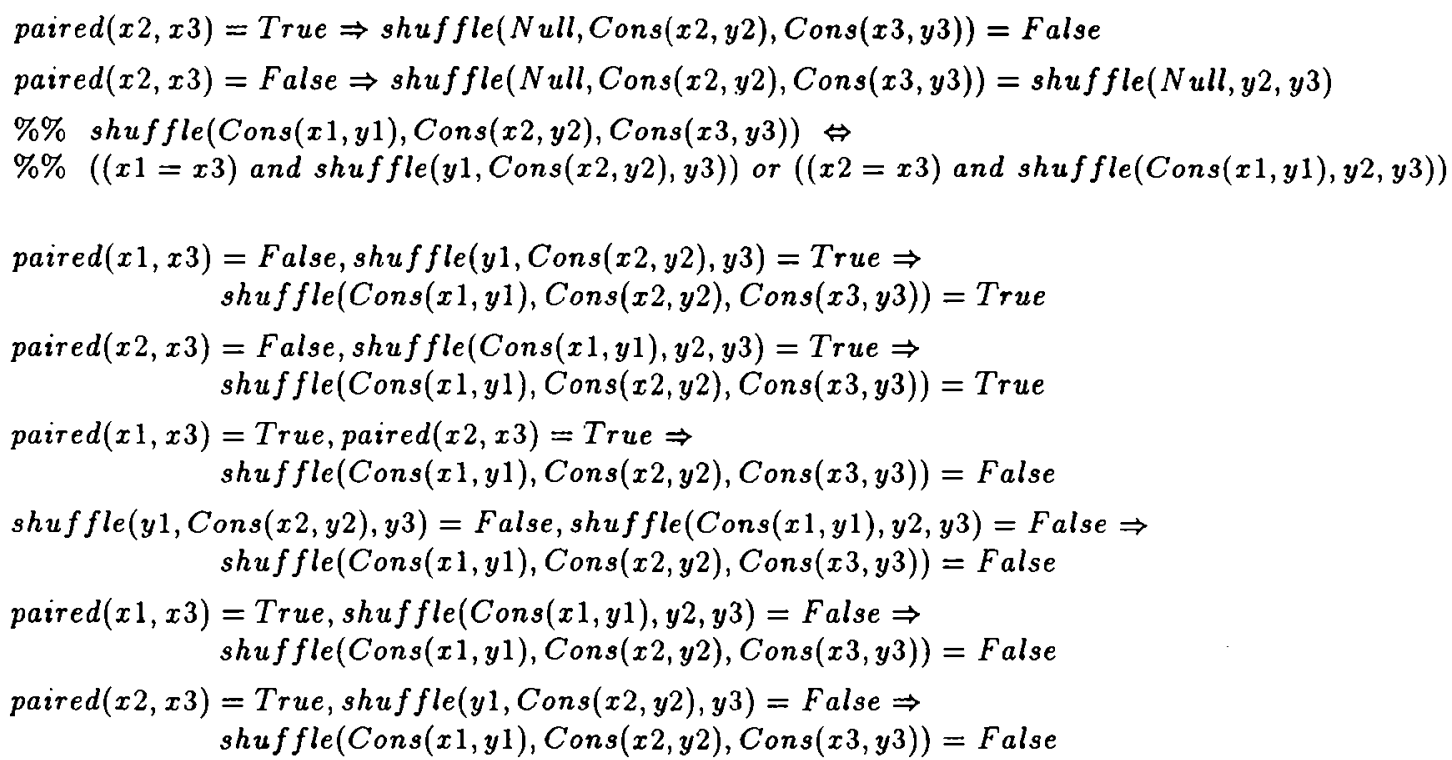

\section{The main theorem}

Imagine $\mathrm{x}$ and $\mathrm{y}$ to be the two card stacks that result from cutting the original deck. The original deck is thus "append $(\mathrm{x}, \mathrm{y})$ ". Suppose that the original deck is of alternating color and of even length. Suppose further that $z$ is a shuffle of $x$ and $y$. If $x$ and $y$ start with cards of opposite color, then $z$ satisfies "pairedlist".

$$
\begin{gathered}
\operatorname{alter}(\operatorname{append}(u, v))=\operatorname{True}, \text { even }(\operatorname{append}(u, v))=\operatorname{True}, \text { opposite }(u, v)=\operatorname{True}, \text { shuffle }(u, v, w)=\operatorname{True} \\
\Rightarrow \text { pairedlist }(w)=\text { True }
\end{gathered}
$$

On the other hand, if $x$ and $y$ start with cards of same color, then the result of moving the top card of $z$ to the end of $z$ satisfies "pairedlist":

$$
\begin{gathered}
\operatorname{alter}(\operatorname{append}(u, v))=\operatorname{True}, \text { even }(\operatorname{append}(u, v))=\operatorname{True}, \text { opposite }(u, v)=\text { False, shuffle }(u, v, w)=\operatorname{True} \\
\Rightarrow \text { pairedlist }(\operatorname{rotate}(w))=\text { True }
\end{gathered}
$$

\section{Properties of the specification}

\section{C.1 Checking the completeness of the specification}

SPIKE checks automatically if an operator $f$ in a specification is completely defined. The program builds a pattern tree for $f$. The leaves of the tree give a partition of the possible arguments for $f$. Then if all the leaves are reducible, the answer is affirmative.

The test is successful on our axioms. This is shown during the first part of the proof session which appears below.

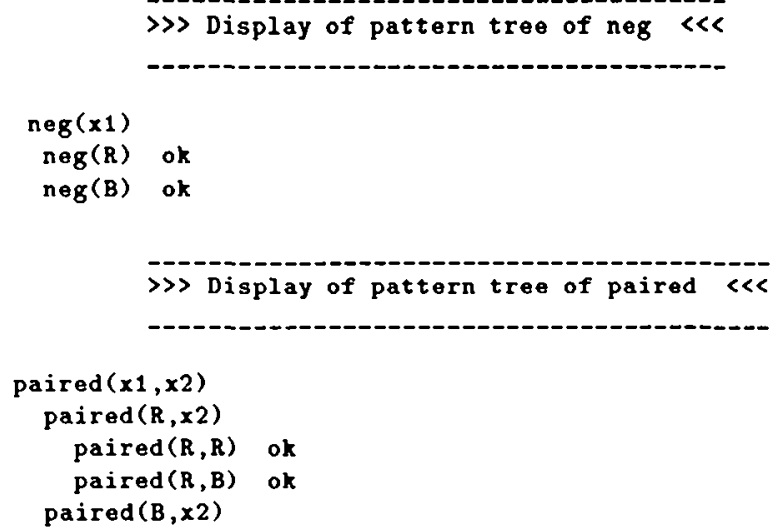


$\operatorname{paired}(B, R)$ ok

paired (B,B) ok

〉 Display of pattern tree of append «<

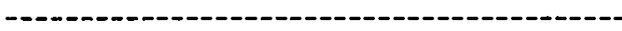

append $(x 1, \times 2)$

append $(\mathrm{Iull}, \times 2)$ ok

append (Cons $(x 3, \times 4), \times 2)$ ok

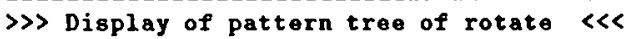

$\operatorname{rotate}(x 1)$

rotate(Bull) ok

rotate(Cons $(\times 2, \times 3))$ ok

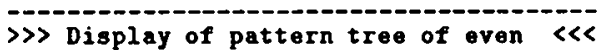

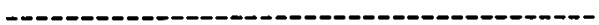

$\operatorname{even}(x 1)$

even(Iull) ok

even (Cons $(x 2, x 3))$

even(Cons ( $x 2$, Iull)) ok

even(Cons $\left(x 2, \operatorname{Cons}\left(x_{1}, x_{4}\right)\right)$ ) ox

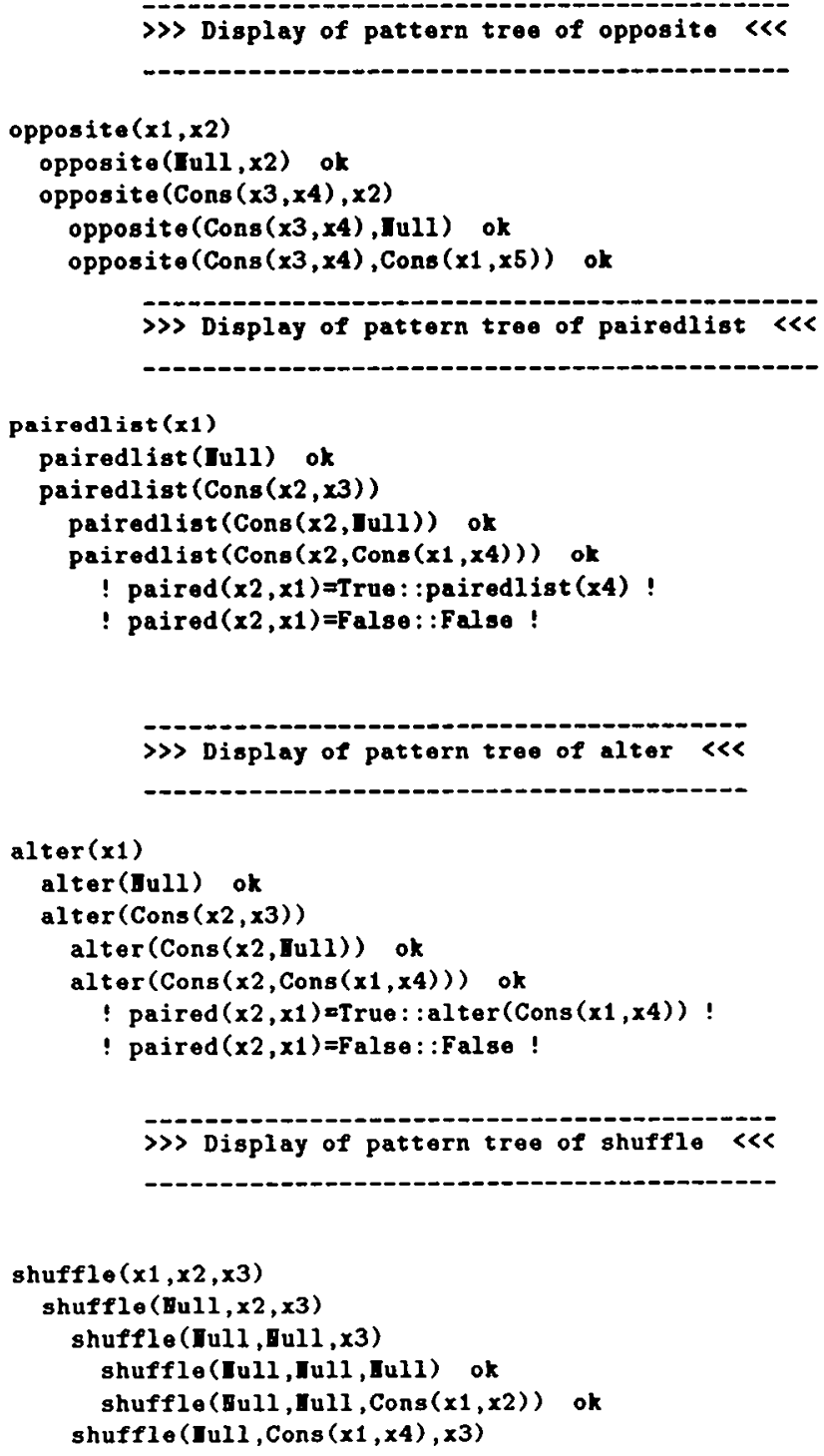




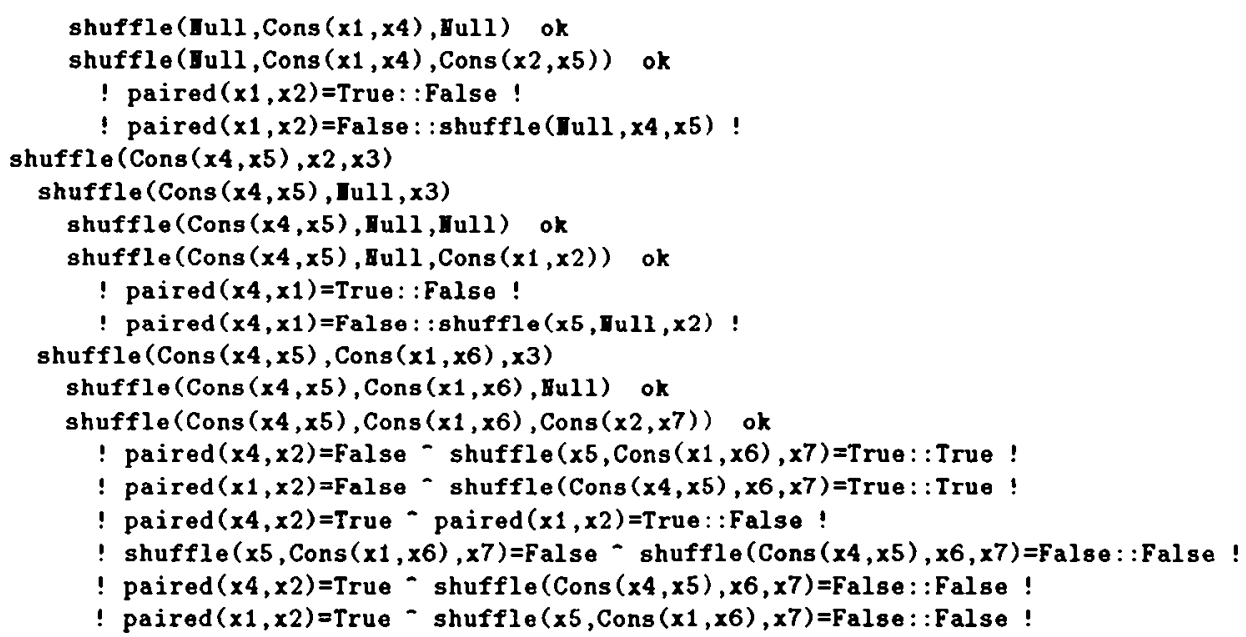

\section{C.2 Check of ground convergence of the specification}

Convergent systems of equations have the property that two terms are equal if and only if they simplify to identical ones. To get convergence, SPIKE uses the saturation technique [Kounalis Rusinowitch 87] which is a natural extension of Knuth and Bendix to conditional theories. This technique is based on a set of inference rules which is refutationally complete for first order logic with equality.

Fragment of the convergence proof is shown. Note that no completion is needed. The initial axioms are already ground convergent.

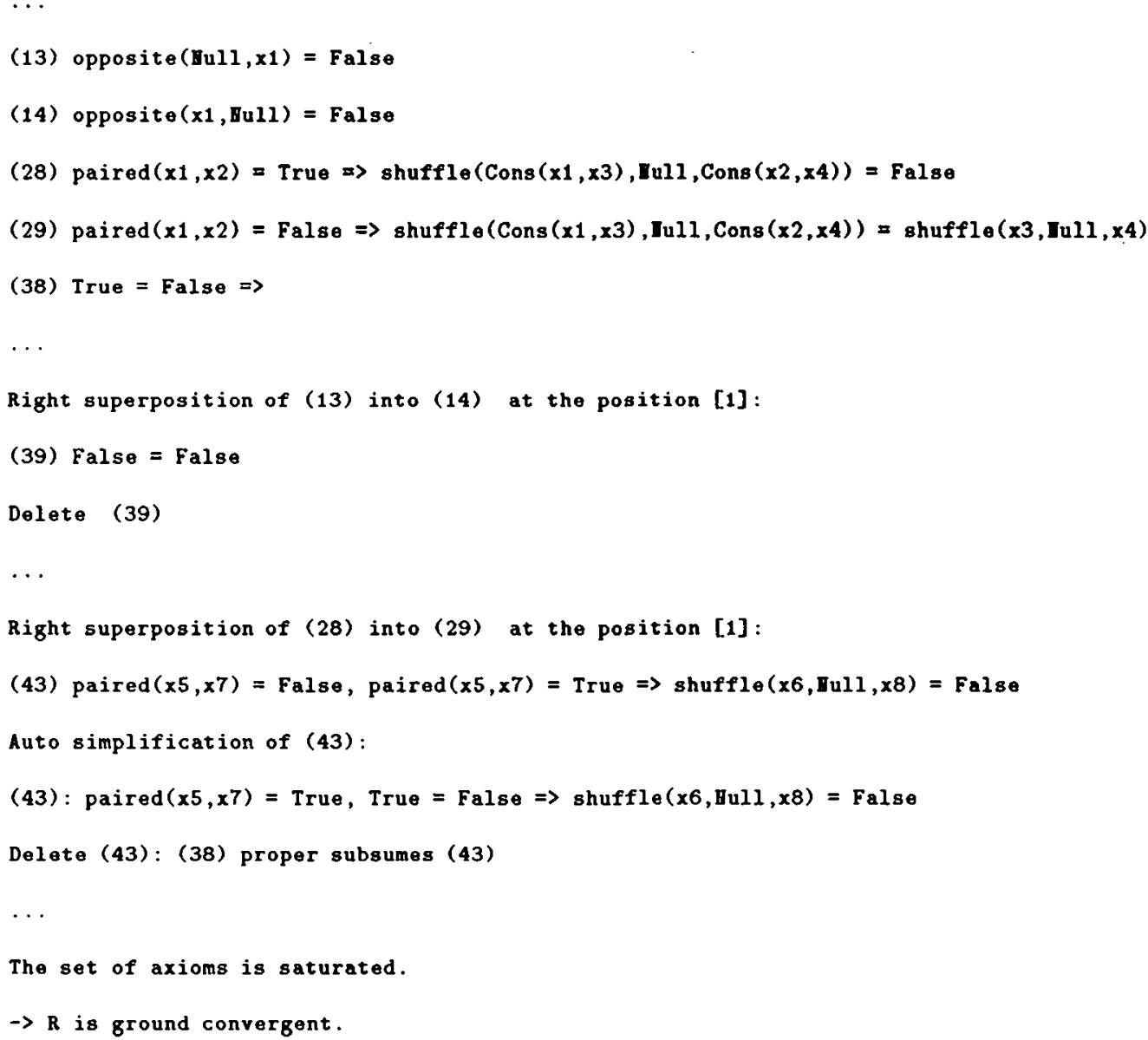




\section{C.3 Computing test sets for $\mathrm{R}$}

Test sets are special induction schemes that ensure that any false boolean conjecture will be detected in finite time, given a convergent specification with completely defined functions.

The output of the SPIKE procedure that computes test sets is:

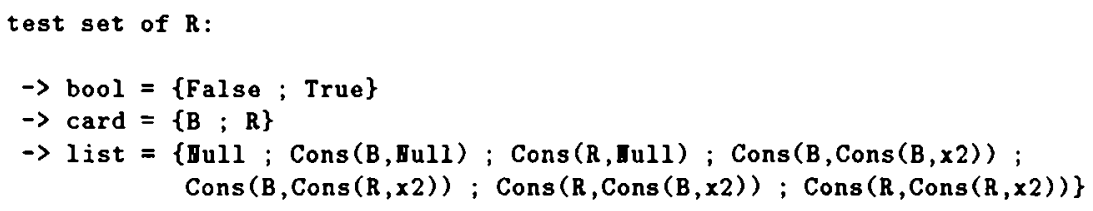

A proof has also been obtained using the following cover sets for the sort list

$$
\{\text { Null, Cons }(x, N u l l), \text { Cons }(x, \text { Cons }(y, z))\}
$$

\section{C.4 Computing inductive positions of functions}

To prove a theorem, it is sufficient to apply induction schemes to variables that occur only at special positions in a term $f\left(x_{1}, \ldots, x_{n}\right)$. This positions are called inductive positions of $f$.

The output of the SPIKE procedure that compute inductive positions of functions is:

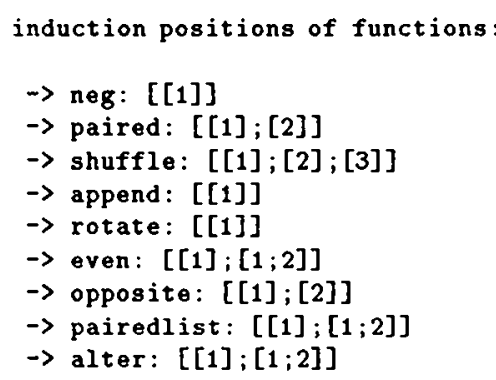

\section{Lemmas}

We provide the system with 5 easy lemmas to help the proof.

Lemma 1 Let $x 1$ and $x 2$ two lists of cards. If $x 1$ is even, then append $(x 1, \operatorname{cons}(x 2, x 3))$ is even iff cons $(x 2, x 3)$ is even.

$\operatorname{even}(x 1)=\operatorname{True} \Rightarrow \operatorname{even}\left(\operatorname{append}\left(x 1, \operatorname{Cons}\left(x_{2}, x_{3}\right)\right)\right)=\operatorname{even}\left(\operatorname{Cons}\left(x_{2}, x_{3}\right)\right)$

Lemma 2 Let $x 1$ and $x 2$ two lists of cards. If $x 1$ is odd, then append( $x 1, \operatorname{cons}(x 2, x 3))$ is even iff $x 3$ is even.

$\operatorname{even}(x 1)=$ False $\Rightarrow \operatorname{even}(\operatorname{append}(x 1, \operatorname{Cons}(x 2, x 3)))=\operatorname{even}(x 3)$

Lemma 3 If Cons $(x 1, y 1)$ is a list of cards whose colors do not alternate then Cons $(x 1$, append $(y 1, y 2))$ is a list of cards whose colors do not alternate either.

$\operatorname{alter}\left(\operatorname{Cons}\left(x_{1}, x_{2}\right)\right)=$ False $\Rightarrow \operatorname{alter}\left(\operatorname{Cons}\left(x_{1}\right.\right.$, append $\left.\left.\left(x_{2}, x_{3}\right)\right)\right)=$ False

Lemma 4 If Cons $(x 1, y 1)$ is a list of cards whose colors alternate and $y 1$ is of odd length, then Cons $(x 1$, append $(y 1, y 2))$ alternate iff Cons $(n \in g(x 1), y 2)$ alternate.

$\operatorname{alter}(\operatorname{Cons}(x 1, x 2))=\operatorname{True}, \operatorname{even}\left(x_{2}\right)=$ False $\Rightarrow \operatorname{alter}(\operatorname{Cons}(x 1$, append $(x 2, x 3)))=\operatorname{alter}\left(\operatorname{Cons}\left(\operatorname{neg}\left(x_{1}\right), x 3\right)\right)$

Lemma 5 If Cons $(x 1, y 1)$ is a list of cards whose colors alternate and $y 1$ is of even length, then Cons $(x 1$, append $(y 1, y 2))$ alternate iff $\operatorname{Cons}(x 1, y 2)$ alternate.

$\operatorname{alter}(\operatorname{Cons}(x 1, x 2))=\operatorname{True}, \operatorname{eren}\left(x_{2}\right)=\operatorname{True} \Rightarrow \operatorname{alter}\left(\operatorname{Cons}\left(x 1, \operatorname{append}\left(x_{2}, x_{3}\right)\right)\right)=\operatorname{alter}(\operatorname{Cons}(\times 1, \times 3))$ 


\section{Remark}

If we formulate the problem as Hantao Zhang in RRL that is with the help of a conditional function "cond", then two lemmas are sufficient for SPIKE to prove Gilbreath card trick, namely:

1. $\operatorname{even}\left(\operatorname{append}\left(x 1, \operatorname{Cons}\left(x_{2}, x_{3}\right)\right)\right)==\operatorname{cond}\left(\operatorname{even}\left(x_{1}\right)=\operatorname{True}, \operatorname{even}(\operatorname{Cons}(x 2, x 3)), \operatorname{even}\left(x_{3}\right)\right)$

2. $\operatorname{arter}(\operatorname{Cons}(x 1$, append $(x 2, x 3)))==$

cond (alter (Cons $(x 1, \times 2))=$ False, False

cond (even $\left(x_{2}\right)=\operatorname{True}, \operatorname{arter}\left(\operatorname{Cons}\left(x_{1}, x_{3}\right)\right)$, alter $\left.\left.\left(\operatorname{Cons}\left(\operatorname{neg}\left(x_{1}\right), x_{3}\right)\right)\right)\right)$

\section{Proof of lemmas}

During a preliminary attempt of a proof, we have noticed that the pattern even(append $(x 1$, Cons $(x 2, x 3))$ ) was a cause of divergence. This has motivated us to introduce lemmas 1 and 2. They allow to replace even (append $(x 1, \operatorname{Cons}(x 2, x 3)))$ by even $(\operatorname{Cons}(x 2, x 3))$ if $x 1$ is even, and $\operatorname{even}(x 3)$ if $x 1$ is odd. In the same way, we eliminate alter(Cons(x1,append(x2,x3))), thanks to lemmas 3,4 and 5 .

The 5 lemmas are proved in a single run. Below, we show partial transcripts of the proof session:

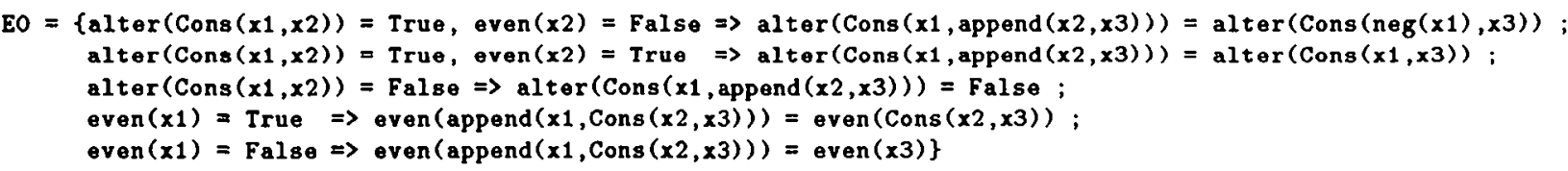

Since in our frameworks an atom is considered to be simpler than its negation, we simplify clauses into positive ones.

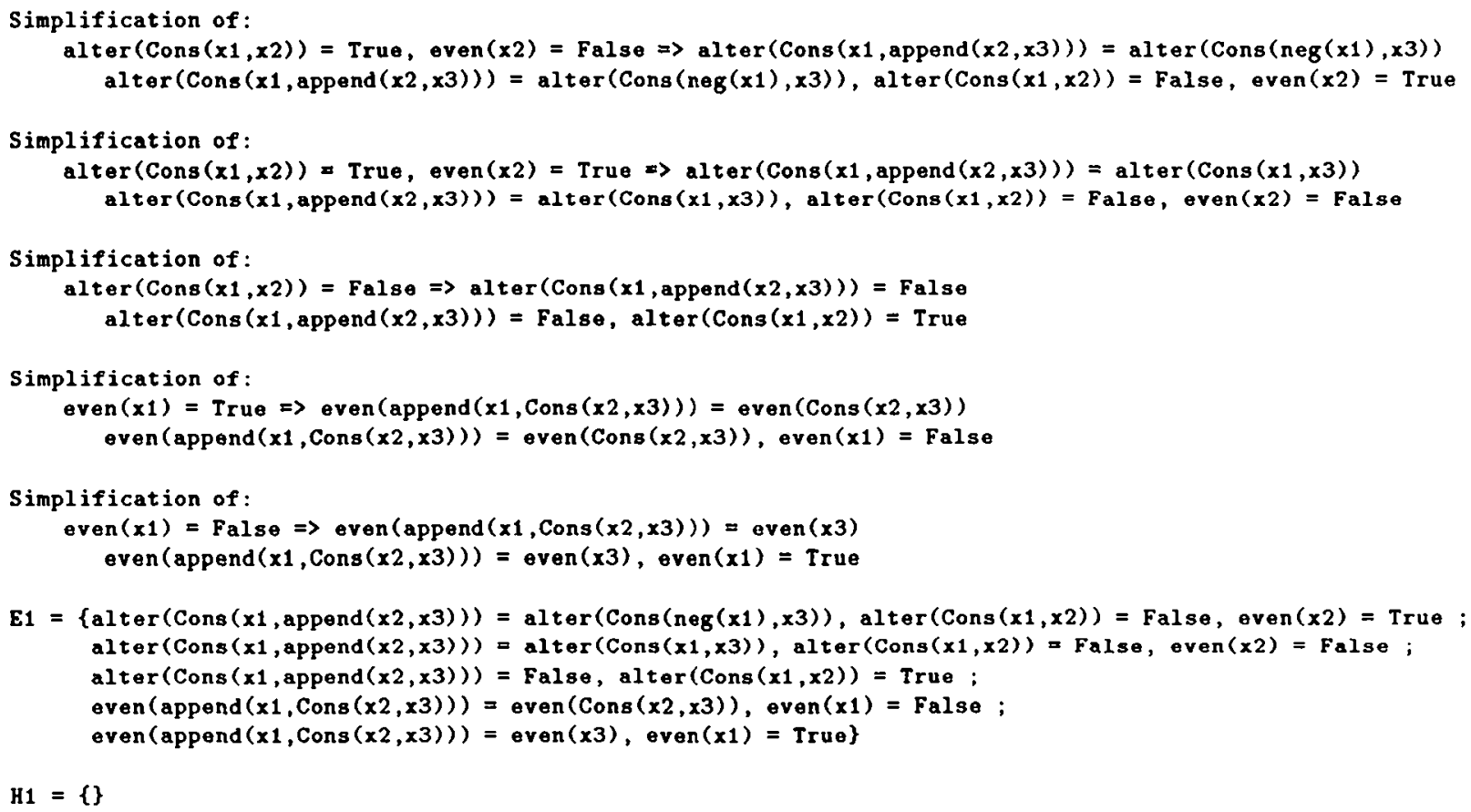

To prove $\operatorname{alter}(\operatorname{Cons}(x 1, \operatorname{append}(x 2, x 3)))=\operatorname{alter}(\operatorname{Cons}(\operatorname{neg}(x 1), x 9)), \operatorname{alter}(\operatorname{Cons}(x 1, x 2))=$ False, even $(x 2)=$ True the induction will be done on $x 1$, and will follow the scheme: 
Application of generate on:

alter(Cons $(x 1, \operatorname{append}(x 2, x 3)))=\operatorname{alter}(\operatorname{Cons}(\operatorname{neg}(x 1), x 3)), \operatorname{alter}(\operatorname{Cons}(x 1, \times 2))=$ False, even $(\times 2)=\operatorname{True}:$

1) $\operatorname{alter}(\operatorname{Cons}(B, x 2))=$ False, $\operatorname{even}(\times 2)=\operatorname{Irue}, \operatorname{alter}(\operatorname{Cons}(B$, append $(x 2, x 3)))=\operatorname{alter}(\operatorname{Cons}(R, x 3))$;

2) $\operatorname{alter}(\operatorname{Cons}(R, \times 2))=\operatorname{False}, \operatorname{even}(\times 2)=\operatorname{True}, \operatorname{alter}(\operatorname{Cons}(R, \operatorname{append}(\times 2, \times 3)))=\operatorname{alter}(\operatorname{Cons}(B, \times 3))$

$E_{2}=\left\{\operatorname{alter}(\operatorname{Cons}(x 1, \operatorname{append}(x 2, x 3)))=\operatorname{alter}(\operatorname{Cons}(x 1, x 3))\right.$, alter $\left(\operatorname{Cons}\left(x 1, x_{2}\right)\right)=$ False, even $\left(x_{2}\right)=$ False ; $\operatorname{alter}(\operatorname{Cons}(x 1, \operatorname{append}(x 2, x 3)))=$ False, alter(Cons $(x 1, x 2))=\operatorname{True}$; even (append $\left(x 1\right.$, Cons $\left.\left.\left(x_{2}, x_{3}\right)\right)\right)=\operatorname{even}\left(\operatorname{Cons}\left(x_{2}, x_{3}\right)\right)$, even $\left(x_{1}\right)=$ False even (append $\left.\left(x 1, \operatorname{Cons}\left(x_{2}, x 3\right)\right)\right)=\operatorname{even}\left(x_{3}\right)$, even $(x 1)=$ True ; $\operatorname{alter}(\operatorname{Cons}(B, x 1))=$ False, $\operatorname{even}(x 1)=\operatorname{True}, \operatorname{alter}(\operatorname{Cons}(B, \operatorname{append}(x 1, \times 2)))=\operatorname{alter}(\operatorname{Cons}(R, x 2))$ $\operatorname{arter}(\operatorname{Cons}(R, x 1))=$ False, $\operatorname{even}(x 1)=\operatorname{True}, \operatorname{alter}(\operatorname{Cons}(R, \operatorname{append}(x 1, \times 2)))=\operatorname{alter}(\operatorname{Cons}(B, x 2))\}$

H2 $=\{\operatorname{alter}(\operatorname{Cons}(x 1, \operatorname{append}(x 2, x 3)))=\operatorname{alter}(\operatorname{Cons}(\operatorname{neg}(\times 1), x 3))$, alter $(\operatorname{Cons}(\times 1, \times 2))=$ False, even $(\times 2)=\operatorname{True}\}$

To prove alter $(\operatorname{Cons}(x 1, \operatorname{append}(x 2, x 3)))=\operatorname{alter}(\operatorname{Cons}(x 1, x 3))$, alter $(\operatorname{Cons}(x 1, x 2))=$ False, even $(x 2)=$ False the induction will be done on $x 2$, and will follow the scheme:

Application of generate on:

$\operatorname{arter}(\operatorname{Cons}(x 1, \operatorname{append}(x 2, x 3)))=\operatorname{alter}(\operatorname{Cons}(x 1, x 3)), \operatorname{alter}(\operatorname{Cons}(x 1, x 2))=$ False, even $(\times 2)=$ False:

1) $\operatorname{arter}(\operatorname{Cons}(x 1, \operatorname{Iul} 1))=$ False, $\operatorname{\theta ven}(\operatorname{Iull})=$ False, alter(Cons $(x 1, x 3))=\operatorname{alter}(\operatorname{Cons}(x 1, x 3))$;

2) $\operatorname{arter}(\operatorname{Cons}(x 1, \operatorname{Cons}(B, \operatorname{Tull})))=$ False,

$\operatorname{even}(\operatorname{Cons}(B, \operatorname{Iu} 11))=\operatorname{False}, \operatorname{alter}(\operatorname{Cons}(x 1, \operatorname{Cons}(B, x 3)))=\operatorname{alter}(\operatorname{Cons}(x 1, x 3)) ;$

3) $\operatorname{alter}(\operatorname{Cons}(x 1, \operatorname{Cons}(R, \operatorname{Iu} 11)))=$ False, even(Cons $(R, I u l 1))=$ False,

alter(Cons $(x 1, \operatorname{Cons}(R, x 3)))=\operatorname{alter}(\operatorname{Cons}(x 1, x 3))$;

4) $\operatorname{alter}(\operatorname{Cons}(x 1, \operatorname{Cons}(B, \operatorname{Cons}(B, \times 2))))=$ False,

even $(\operatorname{Cons}(B, \operatorname{Cons}(B, x 2)))=$ False, alter(Cons $(x 1, \operatorname{Cons}(B, \operatorname{Cons}(B$, append $(x 2, x 3))))=$ alter $(\operatorname{Cons}(x 1, x 3))$;

5) $\operatorname{alter}(\operatorname{Cons}(x 1, \operatorname{Cons}(B, \operatorname{Cons}(R, \times 2))))=$ False,

even(Cons $(B, \operatorname{Cons}(R, x 2)))=$ False, alter(Cons $(x 1, \operatorname{Cons}(B, \operatorname{Cons}(R$, append $(x 2, x 3))))=$ alter $(\operatorname{Cons}(x 1, x 3))$;

6) alter(Cons $(\times 1$, Cons $(R$, Cons $(B, \times 2)))$ ) False,

even(Cons $(R, \operatorname{Cons}(B, x 2)))=\operatorname{False}, \operatorname{alter}(\operatorname{Cons}(x 1, \operatorname{Cons}(R, \operatorname{Cons}(B, \operatorname{append}(x 2, x 3))))=\operatorname{alter}(\operatorname{Cons}(x 1, x 3))$;

7) $\operatorname{alter}(\operatorname{Cons}(x 1, \operatorname{Cons}(R, \operatorname{Cons}(B, \times 2))))=$ False,

even $(\operatorname{Cons}(R, \operatorname{Cons}(R, x 2)))=$ False, alter $(\operatorname{Cons}(x 1, \operatorname{Cons}(R, \operatorname{Cons}(R, \operatorname{append}(x 2, x 3)))))=\operatorname{alter}(\operatorname{Cons}(\times 1, x 3))$

The proof of alter $(\operatorname{Cons}(x 1, \operatorname{Cons}(B, \operatorname{Cons}(B, x 2))))=$ False, even $(x 2)=$ False, alter $(\operatorname{Cons}(x 1, \operatorname{Cons}(B$, $\operatorname{Cons}(B$, append $(x 2, x 3)))))=\operatorname{alter}(\operatorname{Cons}(x 1, x 3))$ is done by a case-analysis on whether paired $(x 1, B)=$ True or paired $(x 1, B)=$ False.

Application of case revriting using $B$ on

alter(Cons $(x 1, \operatorname{Cons}(B, \operatorname{Cons}(B, x 2))))=$ False, even $(x 2)=$ False, $\operatorname{alter}(\operatorname{Cons}(x 1, \operatorname{Cons}(B, \operatorname{Cons}(B, \operatorname{append}(x 2, x 3)))))=\operatorname{arter}(\operatorname{Cons}(x 1, x 3))$ :

1) paired $(x 1, B)=$ True $\Rightarrow$ even $(\times 2)=$ False,

alter $(\operatorname{Cons}(x 1, \operatorname{Cons}(B, \operatorname{Cons}(B, \operatorname{append}(x 2, x 3)))))=\operatorname{arter}(\operatorname{Cons}(x 1, x 3))$, alter $(\operatorname{Cons}(B, \operatorname{Cons}(B, x 2)))=F$ alse ;

2) $\operatorname{paired}(x 1, B)=$ False $\Rightarrow \operatorname{even}\left(x_{2}\right)=$ False,

$\operatorname{alter}(\operatorname{Cons}(x 1, \operatorname{Cons}(B, \operatorname{Cons}(B, \operatorname{append}(x 2, x 3)))))=\operatorname{alter}(\operatorname{Cons}(x 1, x 3))$, False $=$ False; $\ldots$

E60 $=\{\operatorname{aven}(\times 1)=$ False, $\operatorname{alter}(\operatorname{Cons}(B, \operatorname{append}(\times 1, \times 2)))=\operatorname{arter}(\operatorname{Cons}(B, \times 2))$, $\operatorname{alter}($ Cons $(B, \times 1))=$ False, True $=$ False

H60 $=\{\operatorname{alter}(\operatorname{Cons}(x 1, \operatorname{append}(x 2, x 3)))=\operatorname{alter}(\operatorname{Cons}(x 1, x 3))$, alter(Cong $(x 1, x 2))=$ False, even $(x 2)=$ False; $\ldots\}$

Delete even $(x 1)=$ False, alter $(\operatorname{Cons}(B, \operatorname{append}(\times 1, x 2)))=\operatorname{alter}(\operatorname{Cons}(B, x 2))$, $\operatorname{alter}(\operatorname{Cons}(B, x 1))=$ False, $\operatorname{True}=$ False

it is subsumed by:

$\operatorname{alter}(\operatorname{Cons}(x 1, \operatorname{append}(x 2, x 3)))=\operatorname{alter}(\operatorname{Cons}(x 1, x 3)), \quad \operatorname{alter}(\operatorname{Cons}(x 1, x 2))=$ False, even $(\times 2)=$ False of 460

$\mathbf{E 6 1}=\{\}$

The initial conjectures are inductive consequences of $R$

The following sub lemmas have been generated automatically during the proof and have played a role in it: 


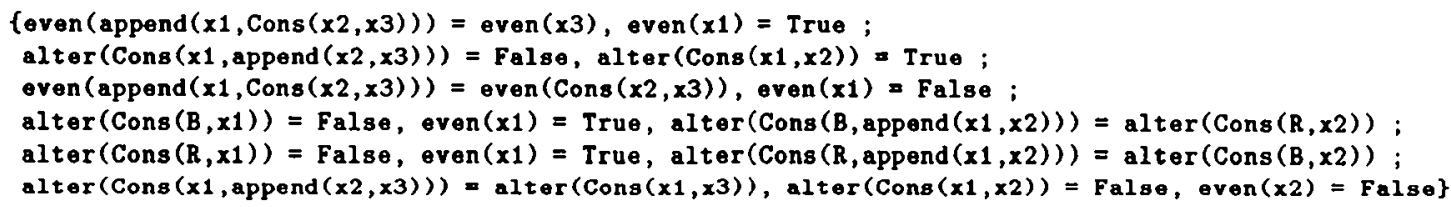

\section{E The main theorem}

Below, we show partial transcripts of the proof session, to illustrate the main steps of the proof:

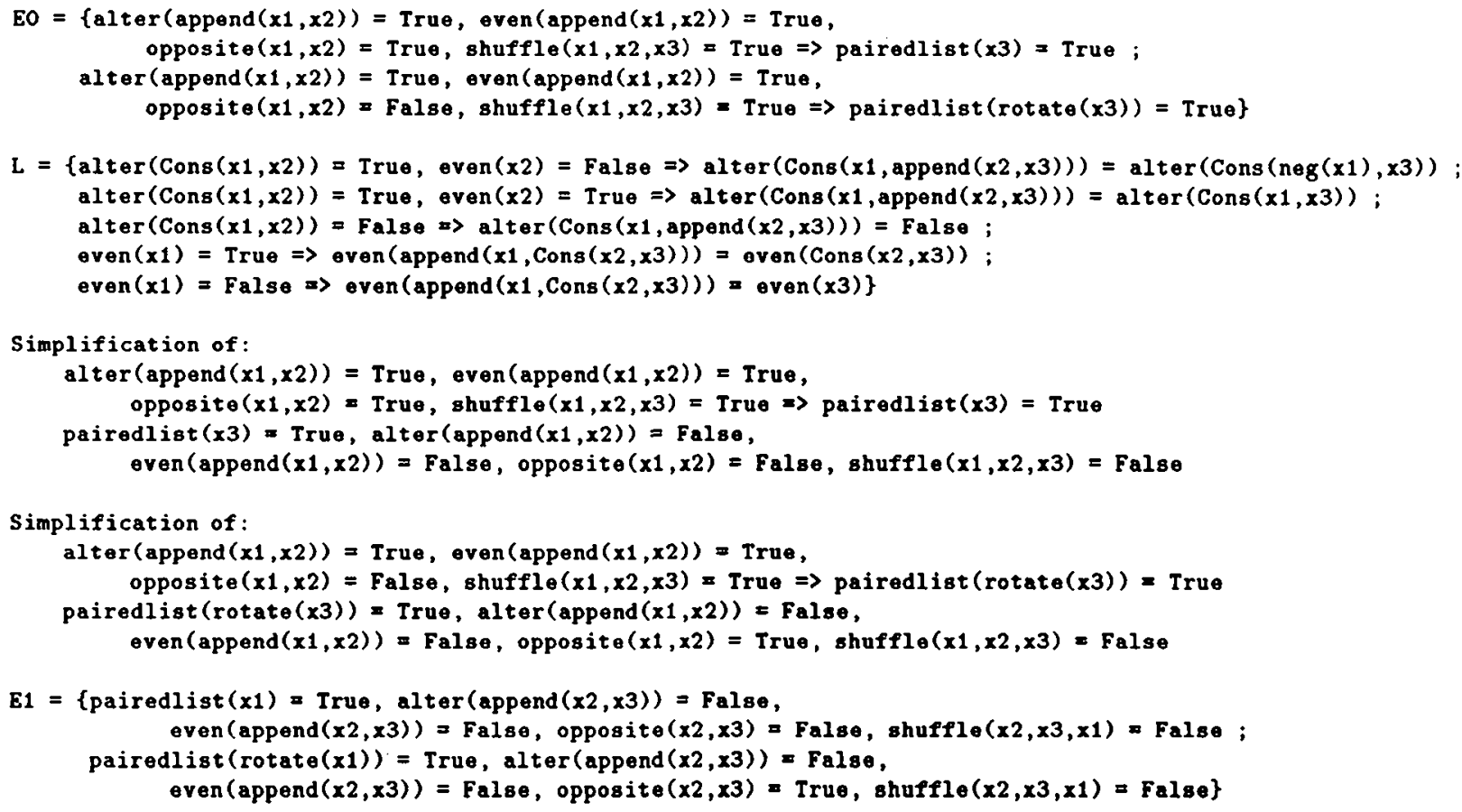

$\mathrm{H} 1=\{\}$

The next case-rewriting step eliminate alter(Cons $(B$, append $(x 1, \operatorname{Cons}(B, \operatorname{Cons}(B, x 2)))))$. Several clauses are generated by this inference step. Some of them are shown below:

Application of case rerriting using $L$ on:

alter(Cons $(B$, append $(x 1$, Cons $(B$, Cons $(B, \times 2)))))=$ False,

even (append $(x 1$, Cons $(B$, Cons $(B, x 2)))$ ) = False, shufflo(Cons $(R, \operatorname{Cons}(B, \times 1)), \operatorname{Cons}(B, \operatorname{Cons}(B, \times 2)), \operatorname{Cons}(B, \operatorname{Cons}(B, \times 3)))=$ False:

1) alter(Cons $(B, x 1))=\operatorname{True}, \operatorname{even}(x 1)=$ False $\Rightarrow$ even (append $(x 1, \operatorname{Cons}(B, \operatorname{Cons}(B, x 2))))=$ False, shuffle (Cons $(R$, Cons $(B, \times 1))$, Cons $(B$, Cons $(B, \times 2))$, Cons $(B$, Cons $(B, \times 3)))=$ False, alter(Cons (neg(B), Cons $(B$, Cons $(B, \times 2)))$ ) = False;

2) alter(Cons $(B, x 1))=\operatorname{True}$, even $(x 1)=\operatorname{True} \Rightarrow \operatorname{even}(\operatorname{append}(x 1, \operatorname{Cons}(B, \operatorname{Cons}(B, \times 2))))=$ False, shuffle(Cons $(R$, Cons $(B, \times 1))$, Cons $(B$, Cons $(B, \times 2))$, Cons $(B, C o n s(B, \times 3)))=$ False, alter(Cons $(B$, Cons $(B$, Cons $(B, \times 2)))$ ) = False ;

3) $\operatorname{alter}(\operatorname{Cons}(B, x 1))=$ False $\Rightarrow$ even (append $(x 1, \operatorname{Cons}(B, \operatorname{Cons}(B, x 2))))=$ False, shuffle $($ Cons $(R$, Cons $(B, \times 1))$, Cons $(B$, Cons $(B, \times 2))$, Cons $(B$, Cons $(B, \times 3)))=$ False, False $=$ False

Application of inductive rewriting to eliminate even(append(x1,Cons $(R, \operatorname{Cons}(B, x 2))))$.

E191 $=$ \{even (append $(x 1, \operatorname{Cons}(R, \operatorname{Cons}(B, x 2))))=$ False,

shuffle (Cons $(R, \operatorname{Cons}(B, x 1))$, Cons $(R, \operatorname{Cons}(B, \times 2)), \operatorname{Cons}(R, \operatorname{Cons}(B, \times 3)))=$ False pairedlist (Cons $(B$, append $(x 3, \operatorname{Cons}(R, B u l 1))))=\operatorname{True}, \operatorname{alter}(\operatorname{Cons}(n e g(B), \operatorname{Cons}(R, \operatorname{Cons}(B, x 2))))=F a l s e$, $\operatorname{alter}($ Cons $(B, \times 1))=$ False, even(x1) = True; 


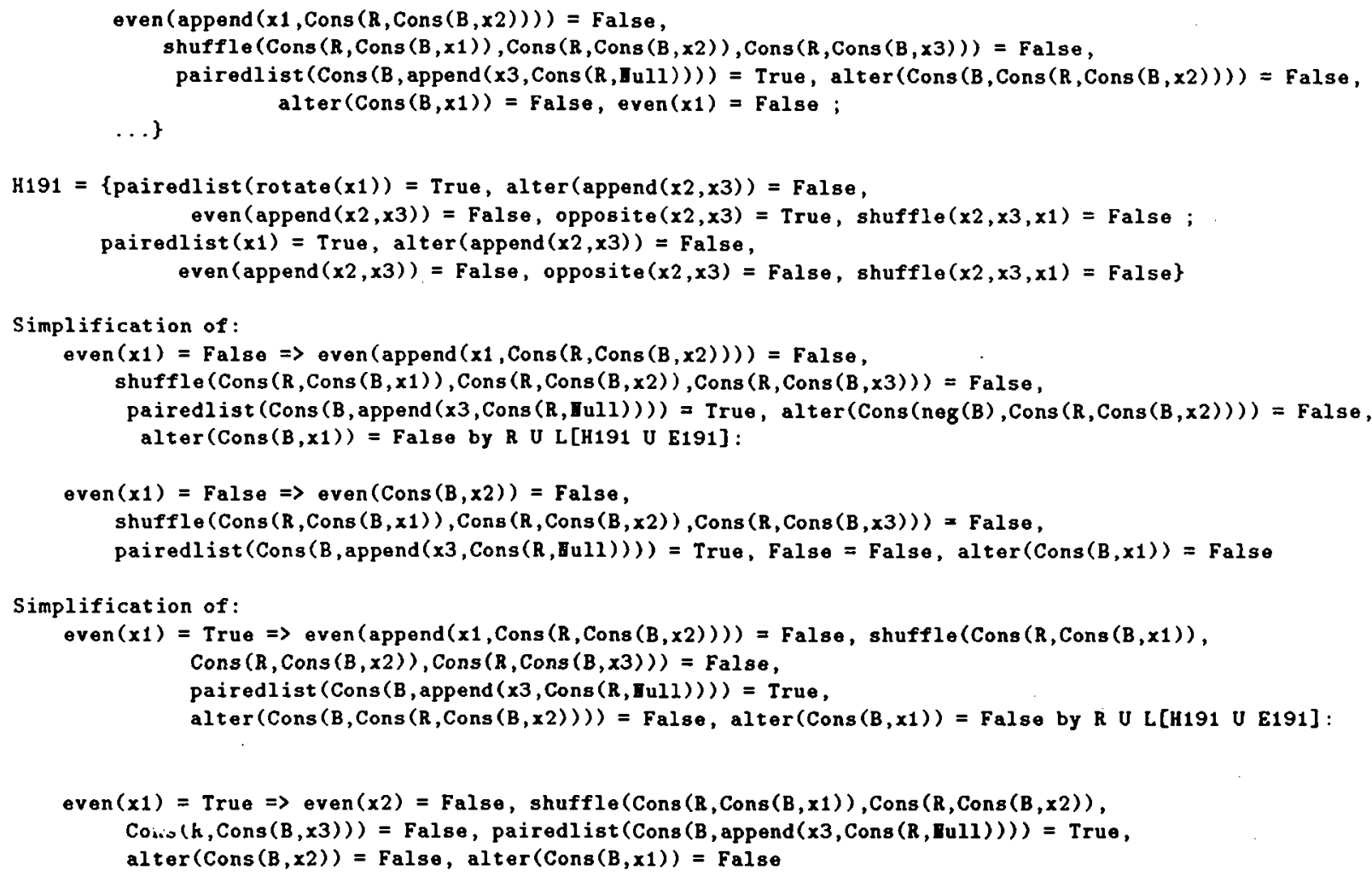

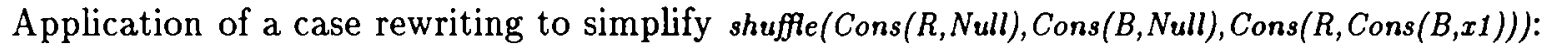

Application of case rerriting using $R$ on:

shuffle(Cons $(R, B u l l), \operatorname{Cons}(B, \operatorname{Bull}), \operatorname{Cons}(R, \operatorname{Cons}(B, \times 1)))=$ False, pairedlist $(x 1)=$ True :

1) $\operatorname{paired}(R, R)=$ False, $\operatorname{shuffle}($ Gull, Cons $(B$, Iull), Cons $(B, \times 1))=$ True $\Rightarrow$ pairedlist(x1) = True, True = False ;

2) $\operatorname{paired}(B, R)=$ False, $\operatorname{shuffle}(\operatorname{Cons}(R, \operatorname{Iull})$, Iull, Cons $(B, x 1))=$ True $\Rightarrow$ pairedlist $(x 1)=\operatorname{True,}$ True $=$ False ;

3) paired $(R, R)=$ True, paired $(B, R)=$ True $\Rightarrow$ pairedlist $(x 1)=$ True, False $=$ False;

4) shuffle $($ Iull, Cons $(B$, Iull), Cons $(B, \times 1))=$ Falsa,

shuffle(Cons (R, Dull), Iull, Cons $(B, x 1))=$ False $\Rightarrow$ pairedlist $(x 1)=$ True, False = False;

5) $\operatorname{paired}(R, R)=$ True, $\operatorname{shuffle}(\operatorname{Cons}(R$, Dull), Dull, Cons $(B, x 1))=$ False $\Rightarrow$ pairedlist $(x 1)=$ True, False $=$ False ;

6) $\operatorname{paired}(B, R)=\operatorname{True}, \operatorname{shuffle}(\operatorname{Iull}, \operatorname{Cons}(B, \operatorname{Iul} 1)$, Cons $(B, \times 1))=$ False $=$ pairedlist (x1) $=$ True, False $=$ False

E912 $=\{\}$

The initial conjectures are inductive consequences of $R$

37 lemmas were generated automatically to prove the conjectures, for example:

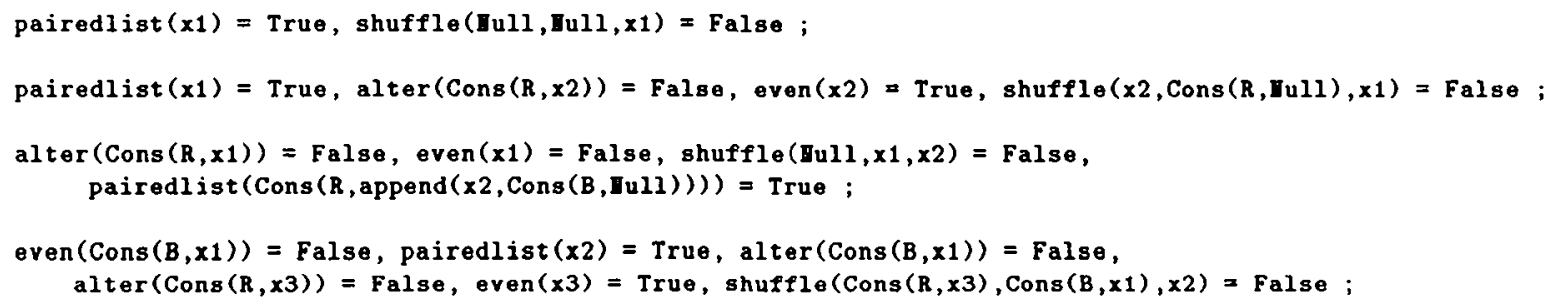

\section{F Refutation of conjectures}

$R$ is a boolean ground convergent rewrite system with completely defined functions. Under these hypotheses SPIKE can refute any false boolean conjecture in finite time. Here is an example of 
a refutation of a false conjecture.

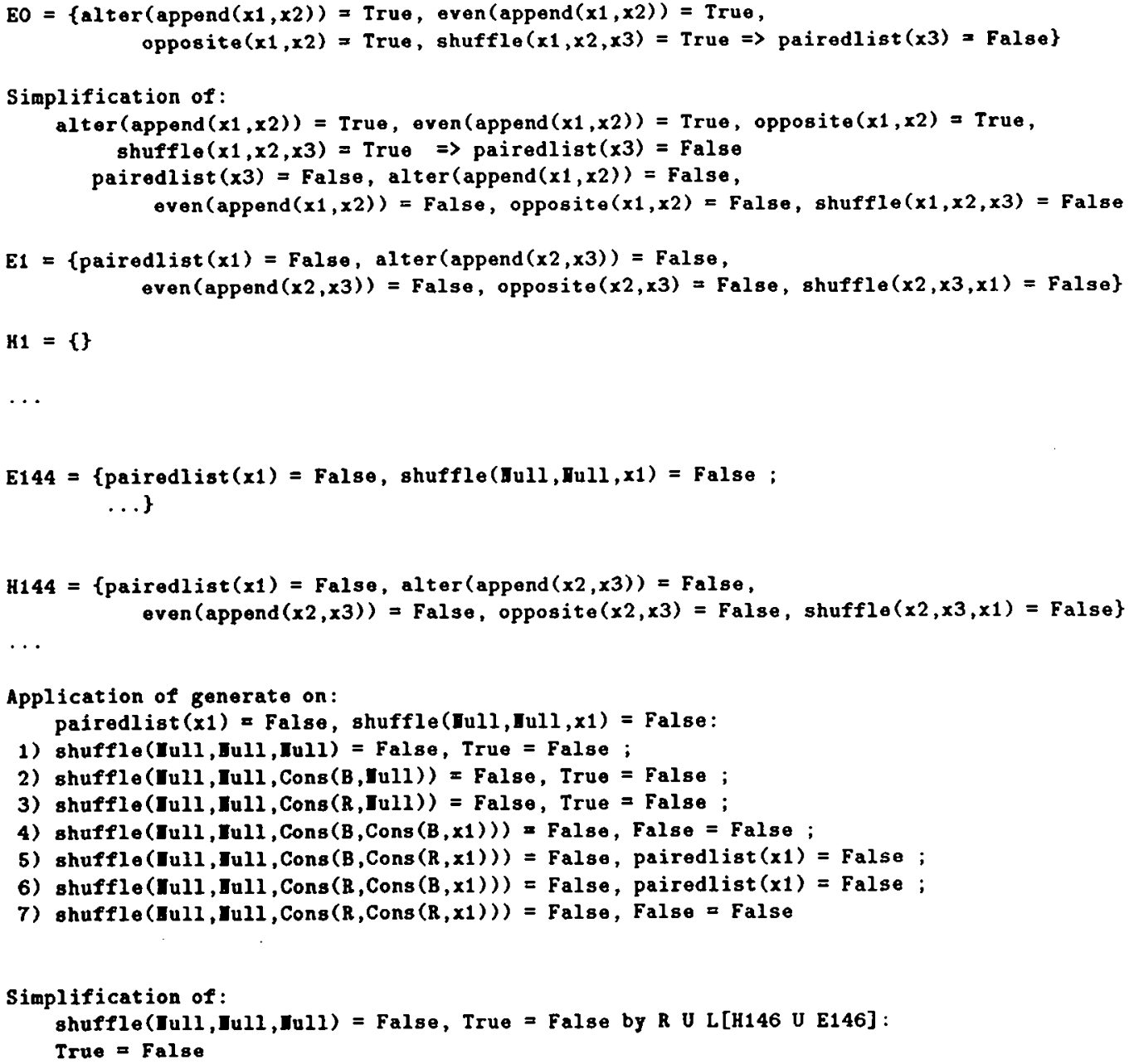

The quasi-inconsistant clause True $=$ False is generated.

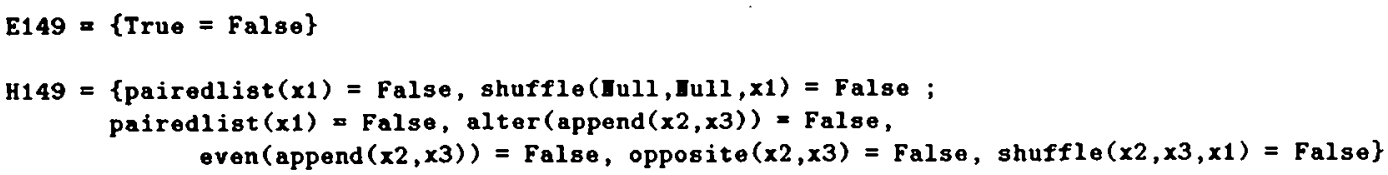

Hence:

The initial conjecture is not an inductive consequence of $R$. 


\section{Appendix 2: Proofs}

Here we prove the lemmas 2 and 4 for the inference system $N$ :

Proof of lemma 2: Let $\Im=\min _{\prec_{c}}\left\{C \sigma / C \in \cup_{i} E_{i}\right.$ and there is a ground substitution $\sigma$ irreducible by $R$ such that $\left.R \nvdash_{\text {ind }} C \sigma\right\}$. $\Im \neq \emptyset$ since $R \nvdash_{\text {ind }} E_{0}$ and $\prec_{c}$ is well-founded. Consider a clause $C \equiv \wedge_{k} a_{k}=b_{k} \Rightarrow \vee_{l} c_{l}=d_{l}$ which is minimal in $\Im$ with respect to the subsumption ordering. It is sufficient to prove that $C$ cannot be simplified nor deleted, and that neither generate nor complement can be applied to $C$; this shows that fail applies since the clause $C$ must not persist in the derivation by the fairness hypothesis.

Hence let us assume that $C \in E_{j}$ and $\left(E_{j}, H_{j}\right) \vdash_{N}\left(E_{j+1}, H_{j+1}\right)$ by some rule applied to $C$. We discuss now the situation according to which rule is applied. In every case we shall derive a contradiction. In order to simplify the notations we write $E$ for $E_{j}, H$ for $H_{j}$ and $P$ for $C \sigma$.

Since $\sigma$ is a ground substitution that is irreducible by $R$, there exists a test substitution $\sigma_{0}$ of $C$ and a ground substitution $\theta$ such that $\sigma=\sigma_{0} \theta$. Before proving the lemma we show the following claim:

\section{claim 1}

1.1 If there is $i_{0}$ such that $C\left[a_{i_{0}}\right] \sim R[H \cup E] ; r C^{\prime}$ where $r$ is the subclause of $C$ such that $C \equiv \neg\left(a_{i_{0}}=b_{i_{0}}\right) \vee r$, then $R F_{\text {ind }} C^{\prime} \sigma$.

1.2 If there is $i_{0}$ such that $C \sigma_{0}\left[a_{i_{0}} \sigma_{0}\right] \sim R[H \cup E] ; r \sigma_{0} C^{\prime}$ where $r$ is the subclause of $C$ such that $C \equiv \neg\left(a_{i_{0}}=b_{i_{0}}\right) \vee r$, then $R \nvdash_{\text {ind }} C^{\prime} \theta$.

1.3 The application of case_rewriting to $C$ generates a clause $C_{k}$ such that $R \nvdash_{\text {ind }} C_{k} \sigma$ and $C_{k} \sigma \prec C \sigma$.

1.4 The application of case_rewriting to $C \sigma_{0}$ generates a clause $C_{k}$ such that $R \forall_{i n d} C_{k} \theta$ and $C_{k} \theta \prec C \sigma$.

proof: We prove only 1.1 and 1.3 since the proof of 1.2 and 1.4 are similar respectively.

1.1 If there exists $i_{0}$ such that $a_{i_{0}} \sim_{R[H \cup E]_{;} r} a_{i_{0}}^{\prime}$, we also have by lemma $1, a_{i_{0}} \sigma \sim_{R[H \cup E]_{; r \sigma}} a_{i_{0}}^{\prime} \sigma$. There are two cases to consider:

If $a_{i_{0}} \sigma \rightarrow_{\text {prem(ro) }} a_{i_{0}}^{\prime} \sigma$, then $R \vDash a_{i_{0}} \sigma=a_{i_{0}}^{\prime} \sigma$ since $R \not \models_{\text {ind }} C \sigma$.

Otherwise, there exists $D \equiv \wedge_{i} a_{i}^{\prime}=b_{i}^{\prime} \Rightarrow c^{\prime} \rightarrow d^{\prime} \in R$ such that: $a_{i_{0}} \sigma \equiv a_{i_{0}} \sigma\left[c^{\prime} \tau\right], a_{i_{0}}^{\prime} \sigma \equiv$ $a_{i_{0}} \sigma\left[d^{\prime} \tau\right]$ and:

i. for all $i$ there exist $e_{i}^{\prime}, e_{i}^{\prime \prime}$ such that: $a_{i}^{\prime} \tau \mapsto_{R \cup H \cup E ; r \sigma}^{*} e_{i}^{\prime}, \quad b_{i}^{\prime} \tau \mapsto_{R \cup H \cup E ; r \sigma}^{*} e_{i}^{\prime \prime}$ and $e_{i}^{\prime}=$ prem(ro) $e_{i}^{\prime \prime}$.

ii. $\left\{a_{i_{0}} \sigma\right\} \gg\left\{a_{1}^{\prime} \tau, b_{1}^{\prime} \tau, \ldots, a_{n}^{\prime} \tau, b_{n}^{\prime} \tau\right\}$.

Let us show that for all $i, R \models a_{i}^{\prime} \tau=b_{i}^{\prime} \tau$ :

We first prove that $a_{i}^{\prime} \tau \mapsto_{R \cup H \cup E ; r \sigma}^{*} e_{i}^{\prime}$ implies $R \vDash a_{i}^{\prime} \tau=e_{i}^{\prime}$ by induction on $a_{i}^{\prime} \tau$ w.r.t. $\succ$. We have $a_{i}^{\prime} \tau \mapsto_{R \cup H \cup E_{; r \sigma}}^{*} e_{i}^{\prime}$. Let $c[g \lambda] \mapsto c[h \lambda]$ be one of the proof step of this derivation.

If $c[g \lambda] \rightarrow_{\text {prem }(r o)} c[h \lambda]$, we have $R \models c[g \lambda]=c[h \lambda]$.

Otherwise, suppose there is $Q \equiv \wedge_{i} g_{i}=h_{i} \Rightarrow g=h \quad \in R \cup H \cup E$ such that:

1. $g \lambda \succ h \lambda$.

2. $\forall i \exists f_{i}^{\prime}, f_{i}^{\prime \prime}$ such that $g_{i} \lambda \mapsto_{R \cup H \cup E_{; r \sigma}}^{*} f_{i}^{\prime}, h_{i} \lambda \mapsto_{R \cup H \cup E_{;} r \sigma}^{*} f_{i}^{\prime \prime}$ and $f_{i}^{\prime}=_{p r e m(r \sigma)} f_{i}^{\prime \prime}$.

3. $c[g \lambda] \succ\left\{g_{1} \lambda, h_{1} \lambda \ldots, g_{n} \lambda, h_{n} \lambda\right\}$.

We have: $g_{i} \lambda \prec c[g \lambda] \preceq a_{i}^{\prime} \tau$, Hence by induction hypothesis we deduce that $R \models g_{i} \lambda=f_{i}^{\prime}$. In the same way $R \models h_{i} \lambda=f_{i}^{\prime \prime}$. On the other side $R \vDash f_{i}^{\prime}=f_{i}^{\prime \prime}$ (since $R \nvdash_{i n d} C \sigma$ ). This implies that for all $i, R \models g_{i} \lambda=h_{i} \lambda$. On the other hand we have $: g_{i} \lambda \prec a_{i}^{\prime} \tau \prec a_{i_{0}} \sigma, h_{i} \lambda \prec a_{i_{0}} \sigma$, 
$g \lambda \preceq a_{i}^{\prime} \tau \prec a_{i_{0}} \sigma$ and $h \lambda \prec g \lambda \prec a_{i_{0}} \sigma$. Hence $Q \lambda \prec{ }_{c} P$ and by hypothesis $Q \lambda$ is valid in $R$. We therefore have $R \models c[g \lambda]=c[h \lambda]$ and as a consequence $R \models a_{i}^{\prime} \tau=e_{i}^{\prime}$.

In the same vein we have $R \models b_{i}^{\prime} \tau=e_{i}^{\prime \prime}$. On the other side $R \models e_{i}^{\prime}=e_{i}^{\prime \prime}$. From the previous results we deduce that for all $i, R \models a_{i}^{\prime} \tau=b_{i}^{\prime} \tau$.

Since $D \in R$ we have $R \models c^{\prime} \tau=d^{\prime} \tau$. This implies that $R \models a_{i_{0}}^{\prime} \sigma=b_{i_{0}} \sigma$ since $R \models a_{i_{0}} \sigma=b_{i_{0}} \sigma$ ( $R \not \models_{\text {ind }} C \sigma$ by hypothesis) and $R \models a_{i_{0}} \sigma=a_{i_{0}}^{\prime} \sigma$ (see above)

Therefore we have $R \forall_{\text {ind }}\left(\wedge_{i \neq i_{0}} a_{i}=b_{i} \wedge a_{i_{0}}^{\prime}=b_{i_{0}} \Rightarrow \vee_{j} c_{j}=d_{j}\right) \sigma$. This shows a contradiction since it proves that we can find an instance of a clause in $E_{j+1}$ which is not valid and smaller than $C \sigma$.

1.3 Assume that the rule case_rewriting can be applied to $C$. Then we have: $C \equiv \neg\left(a_{k}\left[s_{1} \lambda_{1}\right]_{u_{1}}=\right.$ $\left.b_{k}\right) \vee r \equiv \ldots \equiv \neg\left(a_{k}\left[s_{l} \lambda_{l}\right]_{u_{l}}=b_{k}\right) \vee r$ with $\left\{P_{1} \Rightarrow s_{1} \rightarrow t_{1}, \ldots, P_{l} \Rightarrow s_{l} \rightarrow t_{l}\right\} \subseteq R$. The result is: $\left\{C_{1} \equiv \neg\left(a_{k}\left[t_{1} \lambda_{1}\right]_{u_{1}}=b_{k}\right) \vee \neg P_{1} \lambda_{1} \vee r, \ldots, C_{l} \equiv \neg\left(a_{k}\left[t_{l} \lambda_{l}\right]_{u_{l}}=b_{k}\right) \vee \neg P_{l} \lambda_{l} \vee r\right\} \cup C N F\left(P_{1} \lambda_{1} \vee\right.$ $\left.\ldots \vee P_{l} \lambda_{l}\right)$ with $P_{i} \lambda_{i} \ll\left\{s_{i} \lambda_{i}\right\}$ for all $i$. Hence any clause in $C N F\left(P_{1} \lambda_{1} \vee \ldots \vee P_{l} \lambda_{l}\right) \sigma$ is $\ll$ $\left\{a_{k} \sigma\right\}$. This implies that any clause in $C N F\left(P_{1} \lambda_{1} \vee \ldots \vee P_{l} \lambda_{l}\right) \sigma$ is $\prec_{c} P$. As a consequence there exists $i \in[1, \ldots, l]$ such that $R \models$ ind $P_{i} \lambda_{i} \sigma$. Let $C_{i}$ be $\neg\left(a_{k} \sigma\left[t_{i} \lambda_{i}\right]_{u_{i}}=b_{k}\right) \vee \neg P_{i} \lambda_{i} \vee r$. We have $P_{i} \lambda_{i} \sigma \ll\left\{s_{i} \lambda_{i} \sigma\right\}$ and then $P_{i} \lambda_{i} \sigma \ll\left\{a_{k} \sigma\left[s_{i} \lambda_{i} \sigma\right]\right\}$. Since $t_{i} \lambda_{i} \prec s_{i} \lambda_{i}$ we also have $a_{k} \sigma\left[t_{i} \lambda_{i} \sigma\right] \prec a_{k} \sigma\left[s_{i} \lambda_{i} \sigma\right]$. Finally, $C_{i} \prec_{c} P$. On one hand $R \nvdash_{\text {ind }} C \sigma$ and therefore $R \nvdash_{\text {ind }} r \sigma$. On the other hand $R \models_{i n d}\left(P_{i} \Rightarrow s_{i} \rightarrow t_{i}\right) \lambda_{i} \sigma, R \models_{i n d} P_{i} \lambda_{i} \sigma$ and therefore $R \models s_{i} \lambda_{i} \sigma=t_{i} \lambda_{i} \sigma$ and also $R \vDash a_{k} \sigma\left[t_{i} \lambda_{i} \sigma\right]_{u_{i}}=b_{k} \sigma$. Putting everything together we get $R \forall_{\text {ind }} C_{i} \sigma$. A contradiction raises from the generation of a non valid (instance of a ) clause smaller than $C \sigma$. The claim is proved.

We show now that whatever rule is applied to $C$, we obtain a contradiction: generate: There are two cases:

A. generate applies to $C \equiv \neg\left(a_{k}=b_{k}\right) \vee r$. Note that $C \sigma$ cannot be a tautology. We have again two possibilities:

a. there exists $a_{0}$ such that $a_{k} \sigma_{0} \mapsto_{R[H \cup E] ; r \sigma_{0}} a_{0}^{\prime}$. Let $Q \equiv \neg\left(a_{0}^{\prime}=b_{k} \sigma_{0}\right) \vee r \sigma_{0}, Q \in \cup_{i} E_{i}$. From Claim 1.2, $R \nvdash_{\text {ind }} Q \theta$. On the other hand $Q \theta \prec_{c} P$ since $a_{0}^{\prime} \theta=b_{k} \sigma \prec_{e} a_{k} \sigma=$ $b_{k} \sigma$, which is absurd.

b. If case_rewriting applies to $C \sigma_{0}$, then from Claim 1.4, there is a clause $C^{\prime} \in \cup_{i} E_{i}$ with $R \forall_{\text {ind }} C^{\prime} \theta$ and $C^{\prime} \theta \prec_{c} P$. This is also absurd.

B. generate applies to $C \equiv c_{k}=d_{k} \vee r^{\prime}$, This case is similar to A.

case simplify Since case simplification applies to $C$ then by Claim 1.3 there is a clause $C^{\prime} \in \cup_{i} E_{i}$ with $R \nvdash_{\text {ind }} C^{\prime} \sigma$ and $C^{\prime} \sigma \prec_{c} P$, contradiction. The proof is similar whether we use $R$ or $L$ for case simplification.

simplify: If simplify applies to $C$, there are two cases:

A. Simplify applies to $C \equiv \neg\left(a_{k}=b_{k}\right) \vee r$. there are again two possibilities:

A1. $a_{k} \mapsto R[H \cup E] ; r$ a $a^{\prime}$ Let $C^{\prime} \equiv \neg\left(a^{\prime}=b_{k}\right) \vee r$. By Claim $1.1 R \nvdash_{\text {ind }} C^{\prime} \sigma$ and on the other hand $C^{\prime} \sigma \prec_{c} P$, contradiction.

A2. $a_{k} \leadsto H \cup E[R] ; r a^{\prime}$, we also have $a_{k} \sigma \leadsto H \cup E[R] ; r \sigma a a^{\prime} \sigma$. There are two cases:

If $a_{k} \sigma \rightarrow_{\text {prem(ro) }} a^{\prime} \sigma$, then $R \vDash a_{k} \sigma=a^{\prime} \sigma$ since $R \nvdash_{\text {ind }} C \sigma$.

Otherwise, there exists $D \equiv \wedge_{i} s_{i}=t_{i} \Rightarrow s=t \in H \cup E$ such that: $a_{k} \sigma \equiv a_{k} \sigma[s \tau]$ and $a^{\prime} \sigma \equiv a_{k} \sigma[t \tau]$. Let $C^{\prime}$ be $\neg\left(a^{\prime}=b_{k}\right) \vee r$. Then:

i. either $R \vDash s \tau=t \tau$. Then $R \nvdash_{\text {ind }} C^{\prime} \sigma$ and $C^{\prime} \sigma \prec P$, contradiction. 


\section{ii. or $R \not \models s \tau=t \tau$}

Let us show that $R \nvdash_{\text {ind }} C_{s} \tau$. We have:

- $\left\{a_{k} \sigma\right\} \gg\left\{s_{1} \tau, t_{1} \tau, \ldots, s_{n} \tau, t_{n} \tau\right\}$.

- $\forall i \exists e_{i}^{\prime}, e_{i}^{\prime \prime}$ such that : $s_{i} \tau \mapsto_{R \cup H \cup E_{; r \sigma}}^{*} e_{i}^{\prime}, \quad t_{i} \tau \mapsto_{R \cup H \cup E_{;} \sigma \sigma}^{*} e_{i}^{\prime \prime}$ et $e_{i}^{\prime}=_{p r e m(r \sigma)} e_{i}^{\prime \prime}$.

Let us prove that for all $i R \models s_{i} \tau=t_{i} \tau$ : We begin by proving that $R \models s_{i} \tau \sigma=e_{i}^{\prime}$ by induction on $s_{i} \tau \sigma$ w.r.t. $\succ$ : We have $s_{i} \tau \mapsto_{R \cup H \cup E ; r \sigma}^{*} e_{i}^{\prime}$. Let $c[g \lambda] \mapsto c[h \lambda]$ be one of the proof step of this derivation.

If $c[g \lambda] \rightarrow_{\text {prem(ro) }} c[h \lambda]$, we have $R \models c[g \lambda]=c[h \lambda]$.

Otherwise, suppose there is $Q \equiv \wedge_{i} g_{i}=h_{i} \Rightarrow g=h \in H \cup E$ such that :

1. $g \lambda \succ h \lambda$.

2. $\forall i \exists f_{i}^{\prime}, f_{i}^{\prime \prime}$ such that $g_{i} \lambda \mapsto_{R \cup H \cup E ; r \sigma}^{*} f_{i}^{\prime}, h_{i} \lambda \mapsto_{R \cup H \cup E ; r \sigma}^{*} f_{i}^{\prime \prime}$ et $f_{i}^{\prime}={ }_{p r e m(r \sigma)} f_{i}^{\prime \prime}$.

3. $c[g \lambda] \succ\left\{g_{1} \lambda,, h_{1} \lambda \ldots, g_{n} \lambda, h_{n} \lambda\right\}$.

We have: $g_{i} \lambda \prec c[g \lambda] \preceq s_{i} \tau$ and, by induction hypothesis, also: $R \models g_{i} \lambda=f_{i}^{\prime}$. Similarly $h_{i} \lambda \prec s_{i} \tau$ and therefore $R \models h_{i} \lambda=f_{i}^{\prime \prime}$. Since $R \models f_{i}^{\prime}=f_{i}^{\prime \prime}$ which implies that $\forall i R \models$ $g_{i} \lambda=h_{i} \lambda$. We also have: $g_{i} \lambda \preceq s_{i} \tau \prec a_{k} \sigma, h_{i} \lambda \prec s_{i} \tau \prec a_{k} \sigma, g \lambda \prec a_{k} \sigma$ and $h \lambda \prec a_{k} \sigma$. Hence $Q \lambda \prec_{c} P$ and $R \models c[g \lambda]=c[h \lambda]$. It follows that $R \vDash s_{i} \tau=e_{i}^{\prime}$. In the same way we can verify that $R \models t_{i} \tau=e_{i}^{\prime \prime}$. On one hand, $R \models e_{i}^{\prime}=e_{i}^{\prime \prime}$, hence $\forall i R \models s_{i} \tau=t_{i} \tau$, on the other hand $R \not \models s \tau=t \tau \sigma$ hence $R \not \forall_{\text {ind }} C_{s} \tau$. This yields the contradiction since $C_{s} \in \cup_{i} E_{i}$ and $C_{s} \tau \prec_{c} P$.

B. the case where simplify applies to $C \equiv c_{k}=d_{k} \vee r^{\prime}$ is similar to A.

right simplify of constructors: Assume that the rule right simplify of constructors applies to $C \equiv f\left(s_{1}, \ldots, s_{n}\right)=f\left(t_{1}, \ldots, t_{n}\right) \vee r$. Then we have $R \not \forall f\left(s_{1}, \ldots, s_{n}\right) \sigma=f\left(t_{1}, \ldots, t_{n}\right) \sigma$. Since $f$ is a free constructor there exists $i$ such that $R \forall s_{i} \sigma=t_{i} \sigma$. Let $Q \equiv s_{i}=t_{i} \vee r$. Note that $Q \in \cup_{i} E_{i}, R \nvdash_{\text {ind }} Q \sigma$ and $Q \sigma \prec_{c} P$, contradiction.

left simplify of constructors: Assume that the rule left simplify of constructors applies to $C \equiv \neg\left(f\left(s_{1}, \ldots, s_{n}\right)=f\left(t_{1}, \ldots, t_{n}\right)\right) \vee r$. Then we have $R \vDash f\left(s_{1}, \ldots, s_{n}\right) \sigma=f\left(t_{1}, \ldots, t_{n}\right) \sigma$. Since $f$ is a free constructor for all $i, R \models s_{i} \sigma=t_{i} \sigma$. Let $Q \equiv \vee_{i} \neg\left(s_{i}=t_{i}\right) \vee r$. Then $Q \in \cup_{i} E_{i}$, $R \nvdash_{\text {ind }} Q \sigma$ and $Q \sigma \prec_{c} P$, contradiction.

deletion of a trivial clause: Since $R \forall_{\text {ind }} C \sigma, C$ is not a tautology and this rule need not be considered.

subsumption: Since $R \forall_{\text {ind }} C \sigma, C$ cannot be subsumed by an axiom from $R$. If there is $C^{\prime} \in H \cup(E \backslash\{C\})$ such that $C \equiv C^{\prime} \tau \vee r$, we have $R \nvdash_{\text {ind }} C^{\prime} \tau \sigma$ so $r=\emptyset$ and $\tau=\mathcal{I}$ since $C$ is minimal in $\Im$ w.r.t. the subsumption ordering. As a consequence $C^{\prime} \notin(E \backslash\{C\})$. Assume that $C^{\prime} \in H$. Hence generate has been applied to $C^{\prime}$. Therefore generate can be also applied to $C$ in contradiction with a previous case.

complement: Assume that $C \equiv \neg\left(a_{k} \tau=b_{k} \tau\right) \vee r$. Complement generate $Q \equiv a_{k} \tau=b^{\prime} \tau \vee r$. But $R \not \forall\left(a_{k} \tau\right) \sigma=\left(b^{\prime} \tau\right) \sigma$ since $\neg\left(b_{k}=b^{\prime}\right) \in R$. Hence $R \not \nvdash_{\text {ind }} Q \sigma$. Either we have $b^{\prime} \tau \prec b_{k} \tau$ and also $Q \sigma \prec_{c} P$, absurd. Or $b^{\prime} \tau \approx b_{k} \tau$ and $Q \sigma \prec_{c} P$ again since $n \ln (Q \sigma)=n \ln (C \sigma)-1$. This is also impossible.

Proof of lemma 4: Let $R$ be a convergent rewrite system such that all defined symbols are completely defined. Let $C$ be the clause $\wedge_{i} a_{i}=b_{i} \Rightarrow \vee_{j} c_{j}=d_{j} . \in E_{j}$ and assume that $\left(E_{j}, H_{j}\right) \vdash_{I}\left(E_{j+1}, H_{j+1}\right)$ by application of an inference rule on $C$. Let us show that $\forall i \leq$ $j R \models_{\text {ind }} E_{i}$ implies $R \models_{\text {ind }} E_{j+1}$.

Let us first prove the following claim: 


\section{claim 2}

2.1 if there exists $i_{0}$ such that $C\left[a_{i_{0}}\right] \sim C_{s}\left[R \cup H_{j} \cup E_{j}\right] ; r C^{\prime}$ where $r$ is the subclause of $C$ such that $C \equiv \neg\left(a_{i_{0}}=b_{i_{0}}\right) \vee r$ and $C_{s} \in R \cup H_{j} \cup E_{j}$. then $R \vDash_{\text {ind }} C^{\prime}$.

2.2 if case_rewriting applies to $C$ the derived clauses are inductive consequences of $R$.

\section{proof:}

2.1 If $a_{i_{0}} \sim C_{0}\left[R \cup H, \cup E_{j}\right] ; r \quad a_{i_{0}}^{\prime}$, then let $C^{\prime}$ be the clause $\neg\left(a_{i_{0}}^{\prime}=b_{i_{0}}\right) \vee r$. Consider $C^{\prime} \theta$ a ground instance of $C^{\prime}$. We can assume that for all $i \neq i_{0}, \quad R \vDash a_{i} \theta=b_{i} \theta, R \not \models a_{i_{0}} \theta=b_{i_{0}} \theta$ and for all $j \quad R \not \forall c_{j} \theta=d_{j} \theta$ (otherwise the conclusion is immediately derived). We have $a_{i_{0}} \theta \sim C_{s}\left[R \cup H_{j} \cup E_{j}\right] ; r \theta a_{i_{0}}^{\prime} \theta$. If $a_{i_{0}} \theta \rightarrow_{p r e m}(r \theta) a_{i_{0}}^{\prime} \theta$ then immediately $R \vDash a_{i_{0}} \theta=a_{i_{0}}^{\prime} \theta$.

Otherwise $a_{i_{0}} \theta \equiv a_{i_{0}} \theta\left[c^{\prime} \tau\right], a_{i_{0}}^{\prime} \theta \equiv a_{i_{0}} \theta\left[d^{\prime} \tau\right]$ where $C_{s} \equiv \wedge_{i} a_{i}^{\prime}=b_{i}^{\prime} \Rightarrow c^{\prime}=d^{\prime}$, hence:

1. for all $i$ there exists $e_{i}^{\prime}, e_{i}^{\prime \prime}$ such that $a_{i}^{\prime} \tau \mapsto_{R \cup H_{j} \cup E_{j} ; r \theta}^{*} e_{i}^{\prime}, b_{i}^{\prime} \tau \mapsto_{R \cup H_{j} \cup E_{j} ; r \theta}^{*} e_{i}^{\prime \prime}$ and $e_{i}^{\prime}=_{p r e m(r \theta)}$ $e_{i}^{\prime \prime}$.

2. $a_{i_{0}} \theta \succ\left\{a_{1}^{\prime} \tau, b_{1}^{\prime} \tau, \ldots, a_{n}^{\prime} \tau, b_{n} \tau\right\}$.

Let us show that for all $i R \models a_{i}^{\prime} \tau=b_{i}^{\prime} r$ : We first prove that $a_{i}^{\prime} \tau \mapsto_{R \cup H, \cup E_{j} ; r \theta}^{*} e_{i}^{\prime}$ implies $R \models a_{i}^{\prime} \tau=e_{i}^{\prime}$ by induction on $a_{i}^{\prime} \tau$ w.r.t. $\succ$. Let $c[g \lambda] \mapsto c[h \lambda]$ be one of the proof step of this derivation.

If $c[g \lambda] \rightarrow_{\text {prem }(r \theta)} c[h \lambda]$, we have $R \models c[g \lambda]=c[h \lambda]$.

Otherwise, suppose there is $Q \equiv \wedge_{i} g_{i}=h_{i} \Rightarrow g=h \in R \cup H_{j} \cup E_{j}$ such that:

1. $g \lambda \succ h \lambda$.

2. $\forall i \exists f_{i}^{\prime}, f_{i}^{\prime \prime}$ such that $g_{i} \lambda \mapsto_{R \cup H_{j} \cup E_{j} ; r \theta}^{*} f_{i}^{\prime}, h_{i} \lambda \mapsto_{R \cup H_{j} \cup E_{j} ; r \theta}^{*} f_{i}^{\prime \prime}$ and $f_{i}^{\prime}=_{p r e m(r \theta)} f_{i}^{\prime \prime}$.

3. $c[g \lambda] \succ\left\{g_{1} \lambda,, h_{1} \lambda \ldots, g_{n} \lambda, h_{n} \lambda\right\}$.

We have: $g_{i} \lambda \prec c[g \lambda] \preceq a_{i}^{\prime} \tau$ and by induction hypothesis we conclude that $R \models g_{i} \lambda=f_{i}^{\prime}$. In the same spirit we have $h_{i} \lambda \prec a_{i}^{\prime} \tau$ and $R \vDash h_{i} \lambda=f_{i}^{\prime \prime}$. We also have $R \models f_{i}^{\prime}=f_{i}^{\prime \prime}$. Everything put together we get for all $i R \vDash g_{i} \lambda=h_{i} \lambda$. As a consequence: $R \vDash c[g \lambda]=c[h \lambda]$ since $R \models_{i n d} Q$, and therefore $R \vDash a_{i}^{\prime} \tau=e_{i}$. In the same manner we can check that $R \models b_{i}^{\prime} \tau=e_{i}^{\prime \prime}$ and $R \models e_{i}^{\prime}=e_{i}^{\prime \prime}$. From these remarks we derive for all $i R \models a_{i}^{\prime} \tau=b_{i}^{\prime} \tau$. Hence $R \models c^{\prime} \tau=d^{\prime} \tau$ since $R \models_{i_{\text {ind }}} C_{s}$. This implies successively that $R \models a_{i_{0}} \theta=a_{i_{0}}^{\prime} \theta, R \not a_{i_{0}}^{\prime} \theta=b_{i_{0}} \theta, R \models_{\text {ind }} C^{\prime} \theta$.

2.2 If the case-rewriting rule applies to $C$, then we have: $C \equiv \neg\left(a_{k}\left[s_{1} \lambda_{1}\right]_{u_{1}}=b_{k}\right) \vee r \equiv \ldots \equiv$ $\neg\left(a_{k}\left[s_{l} \lambda_{l}\right]_{u_{l}}=b_{k}\right) \vee r$ with $R^{\prime}$ the following set of rules $\left\{P_{1} \Rightarrow s_{1} \rightarrow t_{1}, \ldots, P_{l} \Rightarrow s_{l} \rightarrow t_{l}\right\} \subseteq R$ et $\forall i s_{i} \lambda_{i}$ is irreducible by $R$ and does not contain induction variables. The derived clauses are: $\left\{C_{1} \equiv \neg\left(a_{k}\left[t_{1} \lambda_{1}\right]_{u_{1}}=b_{k}\right) \vee \neg P_{1} \lambda_{1} \vee r, \ldots, C_{l} \equiv \neg\left(a_{k}\left[t_{l} \lambda_{l}\right]_{u_{l}}=b_{k}\right) \vee \neg P_{l} \lambda_{l} \vee r\right\} \cup C N F\left(P_{1} \lambda_{1} \vee\right.$ $\left.\ldots \vee P_{l} \lambda_{l}\right)$ with $P_{i} \lambda_{i} \ll\left\{s_{i} \lambda_{i}\right\}$ for all $i$. Let us show that $R \models_{i n d} P_{1} \lambda_{1} \vee \ldots \vee P_{l} \lambda_{l}$. Assume that there exists a ground $R$-irreducible substitution $\tau$ such that $R \not P_{1} \lambda_{1} \tau \vee \ldots \vee P_{l} \lambda_{l} \tau$ and consider a term $t \equiv s_{i_{0}} \lambda_{i_{0}}$ such that no proper subterm of $t$ matches the left-hand side of a rule (just take for $t$ a subterm $a_{k}$ occurring at a maximal occurrence $u_{i}$ w.r.t. length). The term $t \tau$ is irreducible at the root since $R \not P_{1} \lambda_{1} \tau \vee \ldots \vee P_{l} \lambda_{l} \tau$. Assume otherwise that there exists a rule $r \in R-R^{\prime}$ with left-hand side $g$ that applies to $t \tau$ and $t \tau=g \sigma$. Note that every non variable position of $g$ is a non variable position of $t$ since $t$ does not contain induction variables. In particular $t$ is not an instance of $g$. Since $g$ is linear we can define a substitution by $\rho(x)=t / w$ for every variable $x$ that occurs at some position $w$ of $g$. We have then $t=g \rho$, in contradiction with the assumption that $R^{\prime}$ contains all the rules whose lhs matches $t$.

If the strict subterms of $t$ do not contain defined operators then $t \tau$ cannot be reduced to a non root position since the constructors are free. This is a contradiction with the fact that all defined symbols are completely defined.

If $t$ contains a strict subterm $s=f\left(t_{1}, \ldots, t_{n}\right)$ with a defined operator $f$ and for all $i$, $t_{i} \in T(C, X)$, then $s$ is strongly irreducible (otherwise $s \tau$ is reducible) and does not contain induction variables (by definition of case rewriting). Hence by proposition 4.1. b) there exists a 
ground instance $t \phi$ of $t$ that is strongly irreducible by $R$. These remarks implies a contradiction since $t \phi$ contains a defined operator and all defined operators are completely defined. As a consequence, $R \vDash_{\text {ind }} P_{1} \lambda_{1} \vee \ldots \vee P_{l} \lambda_{l}$.

Assume that there exists $i$ such that: $R \forall_{\text {ind }} C_{i}$. In other words there is a ground instance $C_{i} \theta$ (we can assume that $C \theta$ is ground without loss of generality) such that: $R \nvdash_{i n d} r \theta$, $R \models a_{k} \theta\left[t_{i} \lambda_{i} \theta\right]_{u_{i}}=b_{k} \theta$ and $R \models_{i n d} P_{i} \lambda_{i} \theta$, so $R \models s_{i} \lambda_{i} \theta=t_{i} \lambda_{i} \theta$. Therefore $R \models a_{k} \theta\left[s_{i} \lambda_{i} \theta\right]=b_{k} \theta$. This implies that $R \nvdash_{\text {ind }} C \theta$, absurd. The claim is proved.

We show now that the application of an inference rule on $C$ generates only inductive consequences of $R$.

generate: There are two cases:

A. generate applies to $C \equiv \neg\left(a_{k_{0}}=b_{k_{0}}\right) \vee r$, Let $\sigma_{0}$ be a $S(R)$-substitution of $C$. If $C \sigma_{0}$ is not a tautology then again there are two cases:

a. If there exists $a_{0}^{\prime}$ such that $a_{k_{0}} \sigma_{0} \mapsto_{R\left[H, \cup E_{\text {, }] ; r \sigma_{0}}\right.} a_{0}^{\prime}$, then by claim $2.1, R \models_{\text {ind }} \neg\left(a_{0}^{\prime}=\right.$ $\left.b_{k_{0}} \sigma_{0}\right) \vee r \sigma_{0}$.

b. if case_rewriting applies to $C \sigma_{0}$ then by claim 2.2 . the resulting clauses are inductive consequences of $R$.

B. case_expand applies to $C \equiv c_{k_{0}^{\prime}}=d_{k_{0}^{\prime}} \vee r^{\prime}$, This case is similar to A.

case simplify: If case simplify applies to $C$ the from claim 2.2, only inductive consequences of $R$ are generated.

simplify: If simplify applies to $C$ then there are two possibilities:

A. Simplify applies to $C \equiv \neg\left(a_{k_{0}}=b_{k_{0}}\right) \vee r$. In this case we have $a_{k_{0}} \sim_{C_{s}}\left[R \cup H, \cup E_{j}\right] ; r \quad a_{0}^{\prime}$ with $R \models_{i n d} C_{s}$. From claim 2.1 we deduce $R \models_{\text {ind }} \neg\left(a_{0}^{\prime}=b_{k_{0}}\right) \vee r$.

B. Simplify applies to $C \equiv c_{k_{0}^{\prime}}=d_{k_{0}^{\prime}} \vee r^{\prime}$. Similar to A.

right simplify of constructors: If right simplify of constructors applies to $C \equiv f\left(s_{1}, \ldots, s_{n}\right)=$ $f\left(t_{1}, \ldots, t_{n}\right) \vee r$. then consider is a ground substitution $\sigma$. Since $R \models_{i n d} C \sigma$ we have either $R \models$ ind $r \sigma$ or $R \vDash f\left(s_{1}, \ldots, s_{n}\right) \sigma=f\left(t_{1}, \ldots, t_{n}\right) \sigma$. The first possibility gives immediately the desired conclusion. Hence let us assume the second one. Since $f$ is a free constructor then $\forall i \quad R \vDash s_{i} \sigma=t_{i} \sigma$. Therefore every clause $Q_{i} \equiv s_{i}=t_{i} \vee r$ verifies $R \models_{i n d} Q_{i} \sigma$.

left simplify of constructors: If left simplify of constructors applies to $C \equiv \neg\left(f\left(s_{1}, \ldots, s_{n}\right)=\right.$ $\left.f\left(t_{1}, \ldots, t_{n}\right)\right) \vee r$. Let $\sigma$ be a ground substitution and let us assume that $R \not f\left(s_{1}, \ldots, s_{n}\right) \sigma$ $=f\left(t_{1}, \ldots, t_{n}\right) \sigma$. Since $R \vDash_{\text {ind }} C \sigma$ we have either $R \models_{\text {ind }} r \sigma$ or $R \not \forall f\left(s_{1}, \ldots, s_{n}\right) \sigma=$ $f\left(t_{1}, \ldots, t_{n}\right) \sigma$. The first possibility gives immediately the desired conclusion. Hence let us assume the second one. Since $f$ is a free constructor there exists $i$ such that $R \not \forall s_{i} \sigma=t_{i} \sigma$. Therefore $R \models\left(\vee_{i} \neg\left(s_{i}=t_{i}\right) \vee r\right) \sigma$.

deletion of a trivial clause and subsumption: If $C$ is deleted then $R \models_{i n d} E_{j+1}$ since $E_{j+1} \subseteq E_{j}$ in this case.

complement: Assume that complement applied to $C \equiv \neg\left(a_{k} \theta=b_{k} \theta\right) \vee r$ gives $C^{\prime} \equiv a_{k} \theta=$ $b^{\prime} \theta \vee r$. Let us show $R \models_{i n d} C^{\prime}$. By contradiction assume that $C^{\prime} \tau$ is a ground instance of $C^{\prime}$ such that $R \nvdash_{\text {ind }} C^{\prime} \tau$. We can also assume that $C \tau$ is ground without loss of generality. Then $R \not F_{\text {ind }} r \tau$ and $R \not \forall a_{k} \theta \tau=b^{\prime} \theta \tau$. But we also have $a_{k}=b_{k} \vee a_{k}=b^{\prime} \in E \cup H$. Therefore $R \models_{\text {ind }} a_{k}=b_{k} \vee a_{k}=b^{\prime}$ and it follows that: $R \models a_{k} \theta \tau=b_{k} \theta \tau$. This imlpies that $R \nvdash_{\text {ind }} C \tau$ which is absurd. 


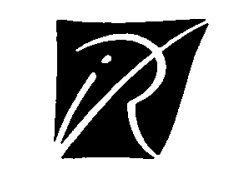

Unité de Recherche INRIA Lorraine

Technopôle de Nancy-Brabois - Campus Scientifique 615, rue du Jardin Botanique - B.P. 101 - 54602 VILLERS LES NANCY Cedex (France)

Unite de Recherche INRIA Rennes IRISA. Campus Lniversitaire de Beaulieu 35042 RFiNNES Cedex (France) linité de Recherche INRIA Rhône-Alpes 46. avenue Félix Viallet - 38031 GRFNOBLE Cédex (France)

Lnité de Recherche INRIA Rocquencourt Domaine de Voluceau - Rocquencourt - B.P. 105 - 78153 L.F. CHESNAY Cedex (France)

Unité de Recherche INRIA Sophia Antipolis 2004, route des Lucioles - B.P. 93-06902 SOPHIA ANTIPOI.IS Cedex (France)

EDITEUR

INRIA - Domaine de Voluceau - Rocquencourt - B.P. 105 - 7815.3 LE CHESNAY Cedex (France)

ISSN $0249-6399$ 